ARTICLE

\title{
Mechanically activated ion channel Piezo1 modulates macrophage polarization and stiffness sensing
}

\author{
Hamza Atcha1,2, Amit Jairaman³, Jesse R. Holt (10 3,4, Vijaykumar S. Meli1,2,5, Raji R. Nagalla (iD) 1,2, \\ Praveen Krishna Veerasubramanian (1) 1,2, Kyle T. Brumm,2, Huy E. Lim ${ }^{1,2}$, Shivashankar Othy ${ }^{3}$, \\ Michael D. Cahalan ${ }^{3}$, Medha M. Pathak (10) ${ }^{1,3,4} \&$ Wendy F. Liu (i) ${ }^{1,2,5 \bowtie}$
}

Macrophages perform diverse functions within tissues during immune responses to pathogens and injury, but molecular mechanisms by which physical properties of the tissue regulate macrophage behavior are less well understood. Here, we examine the role of the mechanically activated cation channel Piezo1 in macrophage polarization and sensing of microenvironmental stiffness. We show that macrophages lacking Piezo1 exhibit reduced inflammation and enhanced wound healing responses. Additionally, macrophages expressing the transgenic $\mathrm{Ca}^{2+}$ reporter, Salsa6f, reveal that $\mathrm{Ca}^{2+}$ influx is dependent on Piezo1, modulated by soluble signals, and enhanced on stiff substrates. Furthermore, stiffnessdependent changes in macrophage function, both in vitro and in response to subcutaneous implantation of biomaterials in vivo, require Piezo1. Finally, we show that positive feedback between Piezo1 and actin drives macrophage activation. Together, our studies reveal that Piezo1 is a mechanosensor of stiffness in macrophages, and that its activity modulates polarization responses.

\footnotetext{
${ }^{1}$ Department of Biomedical Engineering, University of California Irvine, Irvine, USA. ${ }^{2}$ The Edwards Lifesciences Center for Advanced Cardiovascular Technology, University of California Irvine, Irvine, USA. ${ }^{3}$ Department of Physiology and Biophysics, University of California Irvine, Irvine, USA. ${ }^{4}$ Sue and Bill Gross Stem Cell Research Center, University of California Irvine, Irvine, USA. ${ }^{5}$ Department of Chemical and Biomolecular Engineering, University of California

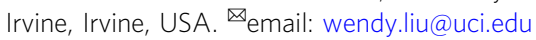


M acrophages are versatile cells of the innate immune system that play critical roles in homeostasis, pathogen defense, and response to injury. This diversity in macrophage function results from their ability to respond dynamically to cues in their microenvironment and polarize towards a multitude of functional phenotypes ${ }^{1-3}$. Pathogens and tissue damage polarize macrophages towards a "classically activated" state that promotes inflammation, whereas wound healing cytokines lead to an "alternatively activated", anti-inflammatory state that aids in tissue repair. In addition to such soluble stimuli, physical cues including tissue stiffness, matrix architecture, and mechanical stimulation are also thought to contribute to macrophage function ${ }^{5-8}$. Our group and others have shown that macrophages cultured on soft substrates $(\sim \mathrm{Pa}-\mathrm{kPa})$ have reduced inflammatory activation when compared to cells adhered to glass or other stiff substrates $(\sim \mathrm{kPa}-\mathrm{GPa})^{5,9,10}$. In addition, mechanical mismatch that occurs when stiff surgical implants are placed within soft tissues has been shown to enhance immune cell recruitment and inflammatory activation ${ }^{11,12}$. Inflammation caused by stiff materials is also associated with a more severe foreign body response and thicker fibrous capsule formation when compared to soft implants, suggesting significant pathological consequences of stiffness-enhanced inflammation ${ }^{10}$. Moreover, increases in tissue stiffness are characteristic of numerous diseases in which macrophages are involved ${ }^{10,13,14}$. While stiffness clearly affects macrophage function, the molecular mechanisms underlying macrophage stiffness sensing are still poorly understood.

Stretch-activated ion channels allow the passage of ions in response to increased membrane tension and play a crucial role in the detection and transduction of external physical stimuli into electrochemical activity that influences signaling and cell behavior ${ }^{15,16}$. A recent flurry of research shows that the mechanically activated, non-specific cation channel Piezol in particular is involved in numerous developmental processes and pathological conditions ${ }^{17-21}$ and it transduces a variety of mechanical cues ${ }^{22-24}$. While increased $\mathrm{Ca}^{2+}$ influx through transient receptor potential (TRP) channels, including TRPM7 and TRPC1, is widely recognized to contribute to macrophage activation by inflammatory agonists ${ }^{25,26}$, the role of Piezol and the $\mathrm{Ca}^{2+}$ signals it generates in response to soluble stimuli remains less well explored. Recent studies have identified Piezol as a mechanosensor of pressure and shear stress in myeloid cells recruited to the lung, heart, and tumors, and found that channel activity stimulates inflammation ${ }^{27-29}$. However, macrophages in most tissues are not subjected to such extreme mechanical stresses, and the mechanical cue that all macrophages are likely to encounter in their environment is variations in matrix stiffness. While Piezol has been shown to sense stiffness in neuronal stem cells and glial cells $s^{17,18}$, a role in macrophage stiffness sensing has not yet been described.

In this study, we investigate the role of Piezol and $\mathrm{Ca}^{2+}$ influx in regulating macrophage responses within varying stiffness environments. We find that Piezol activity promotes interferon- $\gamma(\mathrm{IFN} \gamma)$ and lipopolysaccharide (LPS)-induced inflammatory and suppresses interleukin-4 (IL4) and interleukin-13 (IL13)-induced healing responses. Macrophages expressing the transgenic $\mathrm{Ca}^{2+}$ reporter, Salsa6f, reveal that Piezo1-dependent $\mathrm{Ca}^{2+}$ influx is modulated by soluble signals and increases on stiff substrates. Furthermore, Piezo1 regulates both stiffness-dependent changes in macrophage function in vitro and modulates the immune response to subcutaneous implantation of biomaterials in vivo. Finally, we show that positive feedback between Piezol and actin drives macrophage activation.

\section{Results}

Piezo1 modulates macrophage inflammatory and healing responses. We first evaluated the expression of Piezol in murine bone marrow-derived macrophages (BMDMs) from wild type C57BL/6 J mice, and compared it to the expression of several other channels with known functions in macrophages ${ }^{25,26,30-33}$. Piezol was the most abundantly expressed channel and was more highly expressed than its homolog Piezo2, confirming a recent report (Supplementary Figs. 1 and 2) 27 . In addition, Piezo1 expression increased following stimulation by potent inflammatory agonists, IFN $\gamma$ and LPS (Supplementary Fig. 2). To further explore the role of Piezol in regulating macrophage function, we generated Piezol ${ }^{\text {flox/ }}$ flox ${ }_{\text {LysM }} \mathrm{Cre}^{+}+$mice with Piezol conditionally depleted from LysMexpressing myeloid cells (Piezo1 ${ }^{\Delta L y s M}$ ) and confirmed reduction of Piezol gene expression in isolated BMDMs (Supplementary Fig. 3). We then evaluated the expression of prototypical inflammatory and healing markers in response to in vitro stimulation. We found that BMDMs harvested from Piezo1 ${ }^{\Delta L y s M}$ mice have significantly reduced expression of the inflammatory marker inducible nitric oxide synthase (iNOS) in response to IFN $\gamma / \mathrm{LPS}$ stimulation when compared to BMDMs isolated from control Piezol ${ }^{\text {flox } /+}$ LysM ${ }^{\mathrm{Cre} /+}\left(\right.$ Piezo $\left.^{\mathrm{fl} /+}\right)$ mice (Fig. 1a). In contrast, Piezo1 ${ }^{\Delta L y s M}$ BMDMs exhibited a significant increase in the expression of the healing marker arginase1 (ARG1) in response to wound healing cytokines IL4/IL13, when compared to controls (Fig. 1a). iNOS or ARG1 expression was not observed in unstimulated macrophages, suggesting that Piezo1 expression alone does not polarize macrophages, but it modulates activation by external soluble signals. Examination of cell morphology, which we have previously shown to correlate with activation state $^{34}$, showed that Piezo1 ${ }^{\Delta L y s M}$ BMDMs were more highly elongated compared to control BMDMs in both unstimulated and IFN $\gamma /$ LPS stimulated conditions, whereas cells had similar shape in IL4/ IL13 stimulated conditions (Supplementary Fig. 3). Analysis of other markers of activation, both secreted proteins and gene expression, also showed reduced inflammation and increased healing responses in macrophages lacking Piezo1. Piezo1 ${ }^{\triangle L y s M}$ BMDMs secreted significantly less TNFa and IL6 and had lower Il6 and Nos2 gene expression upon stimulation with IFN $\gamma /$ LPS when compared to control Piezo1 $f l /+$ macrophages (Fig. 1b, c). In addition, IL4/IL13induced Arg1 and Retnla gene expression was higher in Piezo1 ${ }^{\Delta L y s M}$ compared to control BMDMs (Fig. 1c). Resident macrophages isolated from the peritoneum of Piezo ${ }^{\Delta L y s M}$ mice also had suppressed iNOS and enhanced ARG1 expression, similar to what was observed with BMDMs (Supplementary Fig. 4). Together, these studies suggest that Piezol expression enhances inflammatory and reduces wound healing responses in macrophages.

To probe potential downstream mechanisms underlying Piezol-mediated effects, we examined the role of Piezol in regulating transcription factors involved in macrophage activation. Nuclear factor kappa-light-chain-enhancer of activated B cells $(\mathrm{NF \kappa B})$ and signal transducer and activator of transcription 6 (STAT6) are essential transcription factors in inflammatory and healing activation, respectively ${ }^{35-42}$. In addition, the activation of both transcription factors is known to be dependent on $\mathrm{Ca}^{2+}$, where increased intracellular $\mathrm{Ca}^{2+}$ has been shown to enhance NFKB and dampen STAT6 activation 25,43 . We found that cells lacking Piezo1 exhibited reduced IFN $\gamma /$ LPS-induced NFKB activation when compared to control cells (Fig. 1d). IFN $\gamma /$ LPS stimulated Piezo $1^{\triangle L y s M}$ BMDMs also had reduced activation and nuclear localization of STAT1 and STAT3, transcription factors known to play a role in inflammation (Supplementary Fig. 5). On the other hand, IL4/IL13-stimulated BMDMs deficient in Piezol showed increased expression of p-STAT6 (Fig. 1e). These results are consistent with the reduced IFN $\gamma / \mathrm{LPS}$-induced inflammatory and enhanced IL4/IL13-induced healing activation observed in Piezo $1^{\triangle L y s M}$ BMDMs (Fig. 1a-c). Together, these studies suggest that transcriptional regulation of macrophage inflammatory and healing responses are modulated by Piezo1. 
a

b

\section{$f$}
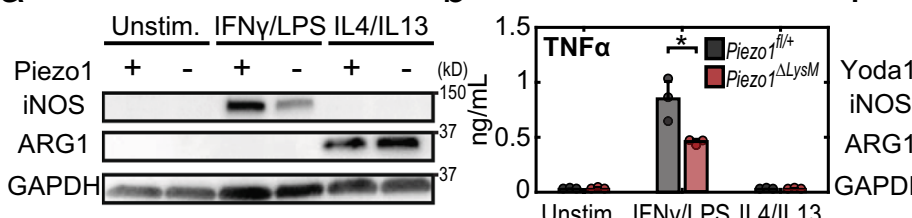

Unstim. IFNy/LPS IL $4 /$ ILL13 9
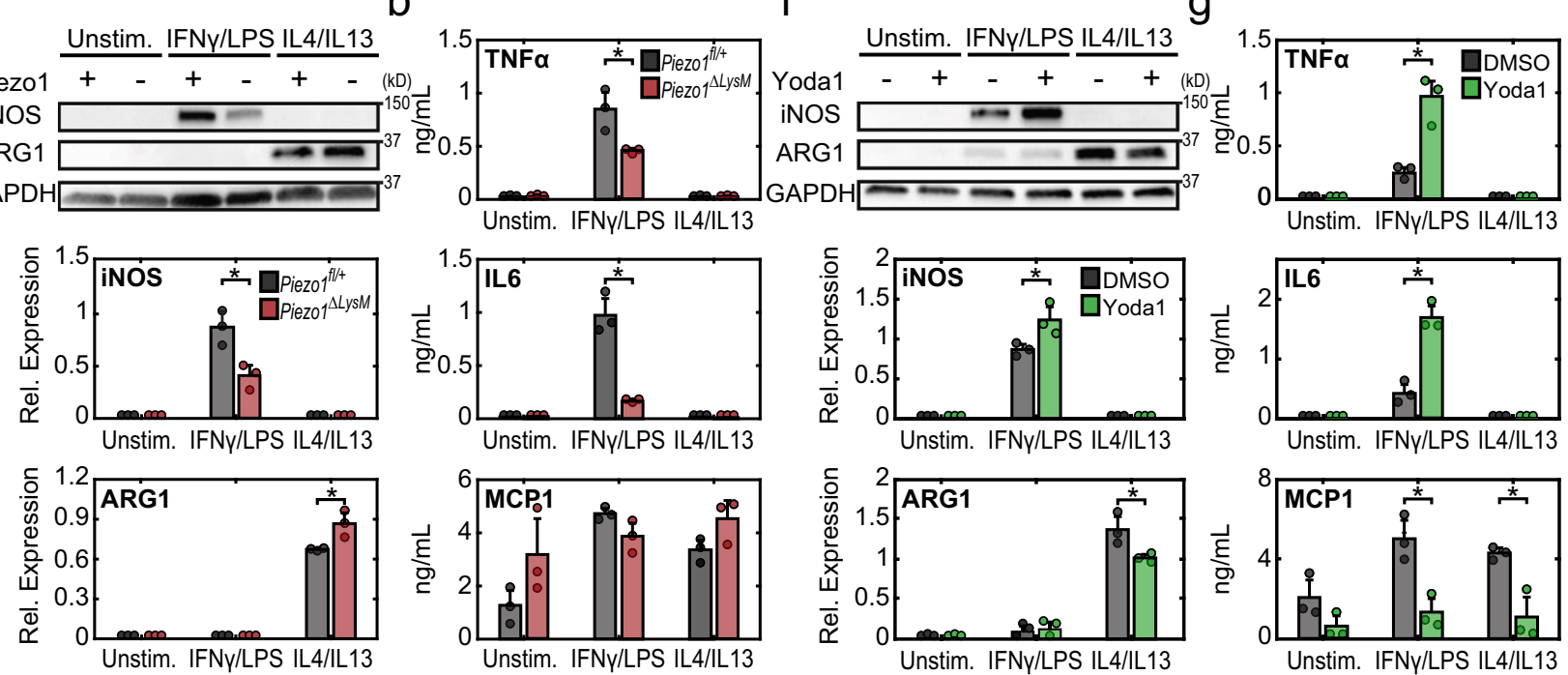

C

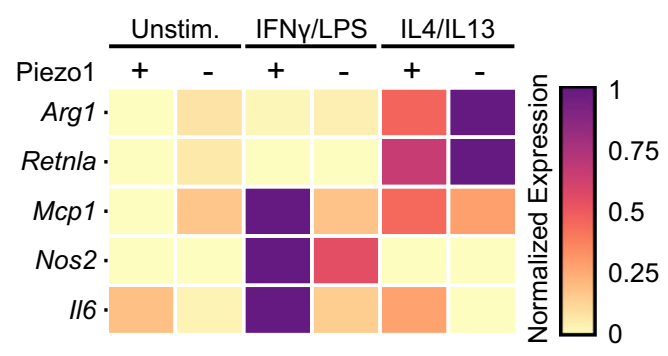

d

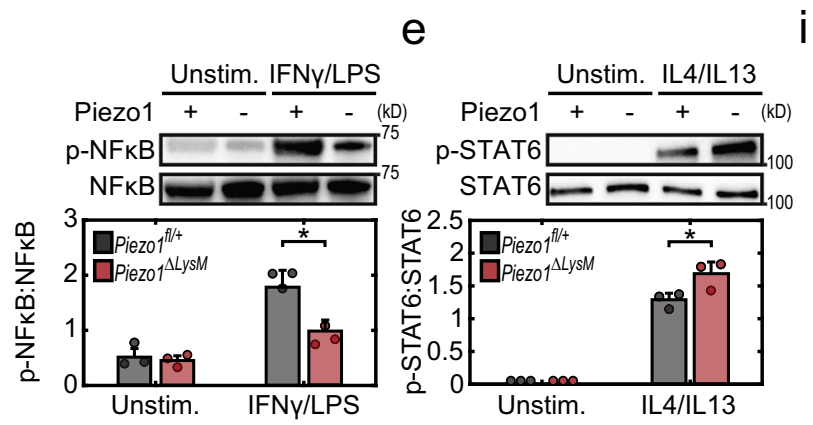

$\mathrm{h}$
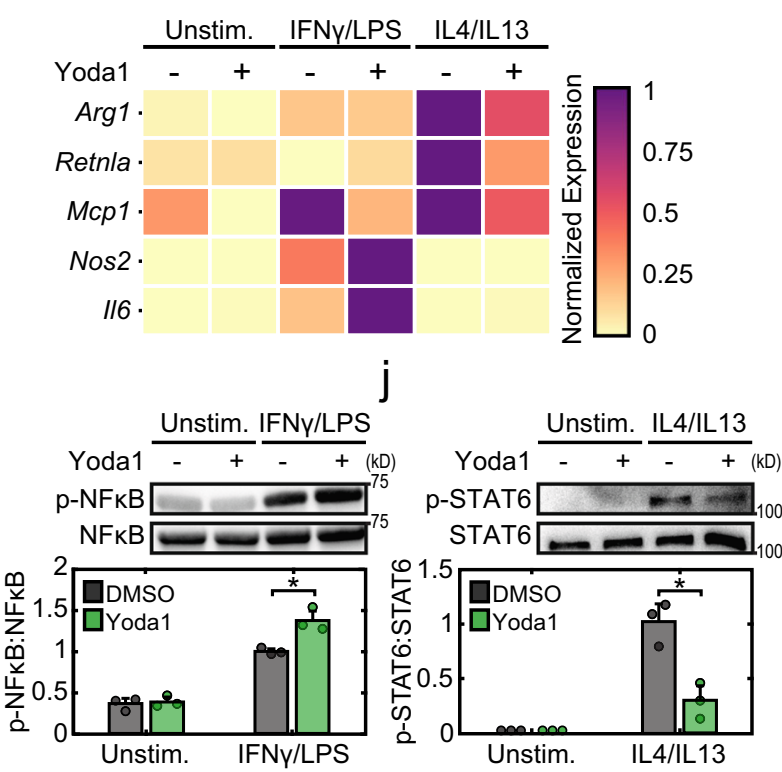

Fig. 1 Piezo1 regulates cytokine-induced macrophage activation. a Representative Western blots (top) and quantification (bottom) of iNOS, ARG1, and GAPDH of Piezo $1^{f l /+}$ and Piezo ${ }^{\Delta L}{ }^{L S M}$ BMDMs incubated with media (Unstim.), IFN $\gamma / \mathrm{LPS}(0.3 \mathrm{ng} / \mathrm{mL}$ of each), or IL4/IL13 (0.1 ng/mL of each). b TNF $\alpha$, IL6, and MCP1 secretion from Piezo $f^{f l /}+$ and Piezo 1 LysM BMDMs incubated with the indicated conditions, as measured by ELISA. c Relative gene expression of inflammatory and healing markers in Piezo $f^{f / /}+$ and Piezo ${ }^{1 L y s M}$ BMDMs incubated with the indicated conditions, as measured by qPCR. Gene expression is shown relative to the highest expressing condition. d, e Representative Western blots (top) and quantification (bottom) of $\mathrm{p}-\mathrm{NFkB} / \mathrm{NFKB}$ of Piezof $f^{f l /+}$ and

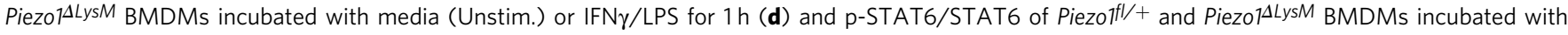
media (Unstim.) or IL4/IL13 for a period of $1 \mathrm{~h}$ (e). f Representative Western blots (top) and quantification (bottom) of iNOS, ARG1, and GAPDH of BMDMs exposed to DMSO or $5 \mu \mathrm{M}$ Yoda1 and stimulated with media (Unstim.), IFN $/ \mathrm{LPS}(0.3 \mathrm{ng} / \mathrm{mL}$ of each), or IL4/IL13 (0.1 ng/mL of each). $\mathbf{g}$ TNF $\alpha$, IL6, and MCP1 secretion from BMDMs exposed to DMSO or $5 \mu \mathrm{M}$ Yoda1 and incubated in the indicated conditions, as measured by ELISA. $\mathbf{h}$ Relative gene expression of inflammatory and healing markers of BMDMs exposed to DMSO or $5 \mu \mathrm{M}$ Yoda1 incubated in the indicated conditions, as measured by qPCR. Gene expression is shown relative to the highest expressing condition. i-j Representative Western blots (top) and quantification (bottom) of $\mathrm{p}-\mathrm{NF} \mathrm{kB} / \mathrm{NF} \mathrm{kB}$ of wild-type BMDMs exposed to DMSO or Yoda1 and stimulated with media (Unstim.) or IFN $\gamma /$ LPS for $2 \mathrm{~h}$ (d) and p-STAT6/STAT6 of Piezoffl/+ and Piezo1 ${ }^{\Delta L y s M}$ BMDMs incubated with media (Unstim.) or IL4/IL13 for a period of $1 \mathrm{~h}$. Error bars denote Mean \pm SD for three independent experiments, ${ }^{\star} p<$ 0.05 as determined by two-tailed Student's $t$ test. Phosphorylated and total forms of transcription factors were obtained from loading equal amounts of protein in separate gels and the resulting blots were processed in parallel. Source data including exact p-values are provided as a Source Data file.

To examine the effects of other modulators of Piezol and ion channel activity, we used siRNA to knock down Piezo1 expression, gadolinium chloride $\left(\mathrm{GdCl}_{3}\right)$ to broadly target ion channels, and GsMTx-4 to target mechanosensitive ion channels. We observed that Piezol siRNA, $\mathrm{GdCl}_{3}$, and GsMTx-4 all generally suppressed both inflammatory responses to IFN $\gamma / \mathrm{LPS}$ and healing responses to IL4/IL13 in BMDMs (Supplementary
Figs. 6, 7), confirming the role of Piezol in inflammation but suggesting that constitutive knockout of this ion channel may be required for upregulating healing responses, and that transient or incomplete siRNA-mediated knockdown as well as non-specific channel inhibition with $\mathrm{GdCl}_{3}$ or GsMTx-4 may not be sufficient. Conversely, to evaluate whether activating Piezo1 influences macrophage function, we utilized a Piezol-specific agonist, 
a
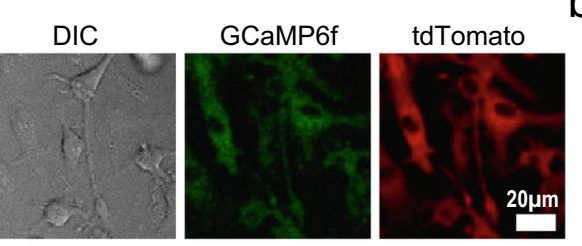

b
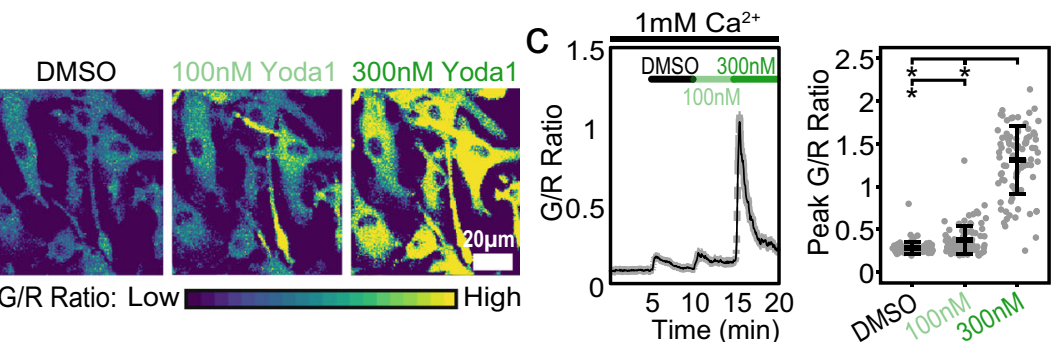

d

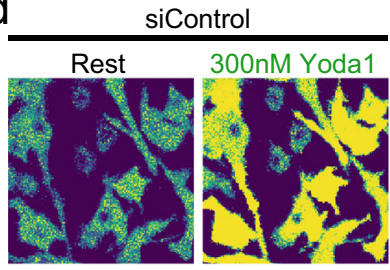

G/R Ratio: Low

f

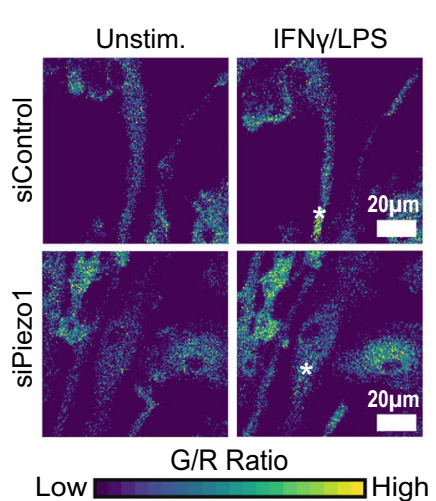

i

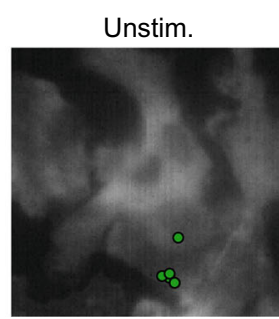

siPiezo1
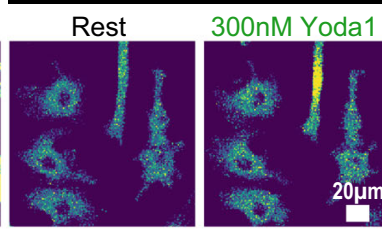

High

g

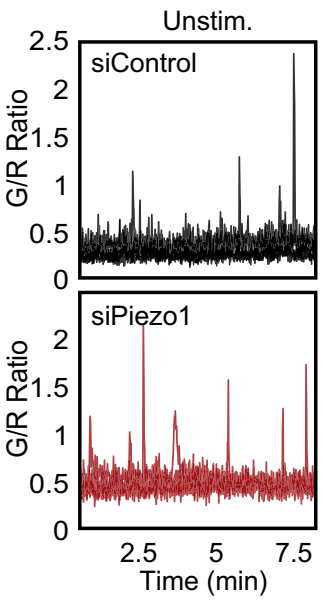

IFNY/LPS

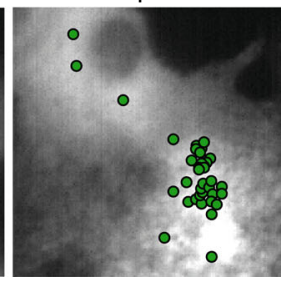

IL4/IL13

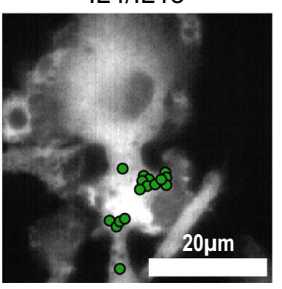

e

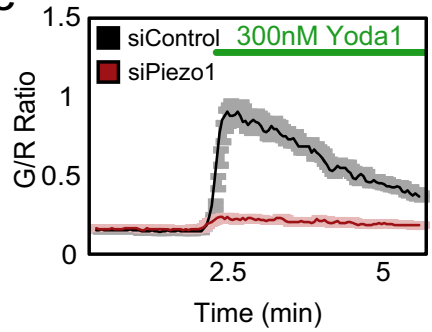

h

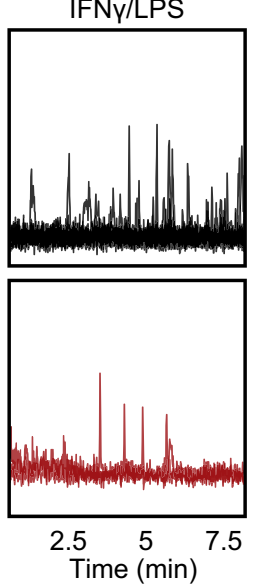

.

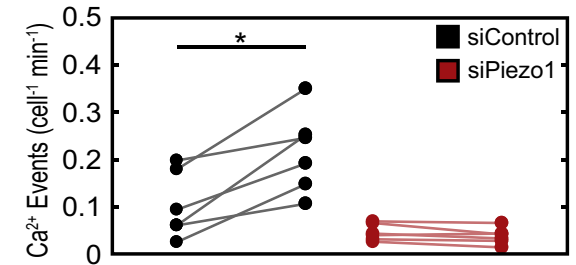

IFNY/LPS:

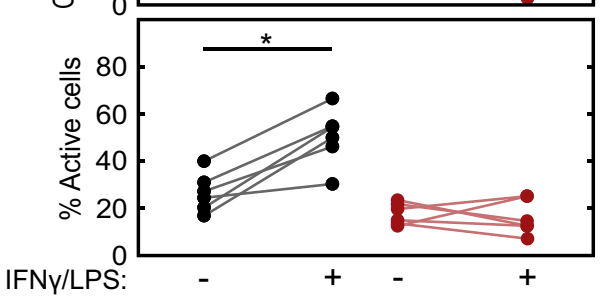

$\mathrm{k}$

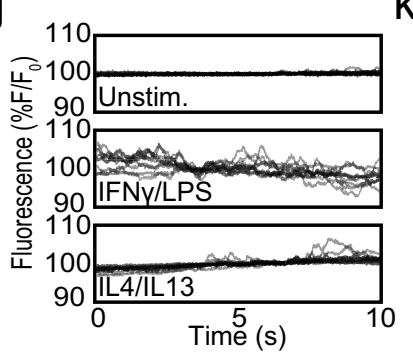

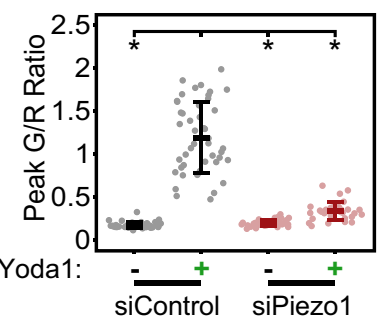

siControl siPiezo1 
Fig. 2 Regulation of $\mathbf{C a}^{2}+$ influx by Piezo1 channels in macrophages. a Representative differential interference contrast (DIC) and fluorescence images showing expression of Salsa6f probe in BMDMs isolated from Vav1-Salsa6f mice, tdTomato is displayed in red and GCaMP6f is displayed in green. $\mathbf{b}$ Green:Red (G/R) ratio images showing $\mathrm{Ca}^{2+}$ responses to Yoda1 in Salsa6f+ BMDMs when exposed to DMSO (black), $100 \mathrm{nM}$ (light green), and $300 \mathrm{nM}$ (dark green) Yoda1, all in $1 \mathrm{mM} \mathrm{Ca}{ }^{2}+$ Ringer solution. c $G / R$ traces averaged across all cells in a field of view over time (left) and quantification of peak $G / R$ ratios per cell (right) of Salsa6f+BMDMs in response to Yoda1. $N=87$ cells, representative of three independent experiments, error bars denote Mean \pm $\mathrm{SD},{ }^{\star} p<0.05$ as determined by two-tailed Mann-Whitney $U$ test). d Representative images showing Ca ${ }^{2+}$ responses to $300 \mathrm{nM}$ Yoda in Salsa6f+ BMDMs treated with either non-target (siControl) or Piezo1 (siPiezo1) siRNA. e G/R traces averaged across all cells in a field of view over time (left) and quantification of peak G/R intensities per cell (right) of siControl and siPiezo1 treated BMDMs exposed to Ringer solution (Rest) or $300 \mathrm{nM}$ Yoda1 in Ringer solution. $N=46$ and 31 cells for siControl and siPiezo1 conditions, representative of three independent experiments, error bars denote Mean \pm SD, * $p<0.05$ as determined by two-tailed Mann-Whitney $U$ test). $\mathbf{f}-\mathbf{h}$ Representative $\mathrm{G} / \mathrm{R}$ ratio images (f), traces of individual $\mathrm{Ca}^{2+}$ events (g), and quantification of number of $\mathrm{Ca}^{2+}$ events (normalized for cell number and time) and fraction of cells showing $\mathrm{Ca}^{2+}$ elevations (h) taken from a time-lapse video of siControl and siPiezo1 treated Salsa6f+ BMDMs following acute addition of Ringer solution (Unstim.) or Ringer solution containing $100 \mathrm{ng} / \mathrm{mL}$ IFN $\gamma /$ LPS. Asterisks denote the occurrence of a $\mathrm{Ca}^{2+}$ event. Each data point in (h) denotes a single video $\left(N=6\right.$ videos, ${ }^{\star} p<0.05$ as determined by twotailed paired $t$ test). $\mathbf{i}-\mathbf{k}$ Representative images overlaid with centroids denoting $\mathrm{Ca}^{2+}$ flickers in green (i) and traces of individual $\mathrm{Ca}^{2+} \mathrm{flickers}(\mathbf{j})$ recorded in unstimulated, $0.3 \mathrm{ng} / \mathrm{mL}$ IFN $/$ LPS, and $0.1 \mathrm{ng} / \mathrm{mL}$ IL4/IL13 stimulated Salsa6f+ BMDMs using high speed TIRF microscopy. $\mathbf{k}$ Frequency of Ca2+ flickers in unstimulated, IFN $/$ LPS, and IL4/IL13 stimulated BMDMs. Each data point represents the frequency of $\mathrm{Ca}^{2+}$ flickers in a single video each composed of one or more cells. $N=21,18$, and 10 fields of view for Unstim., IFN $\gamma /$ LPS and IL4/IL13 conditions, respectively. Error bars denote Mean \pm SD, and ${ }^{\star} p<0.05$ as determined by two-tailed Mann-Whitney $U$ test). Source data including exact $p$-values are provided as a Source Data file.

presence of functional Piezol channels on the cell membrane. Yodal produced a dose-dependent increase in cytosolic $\mathrm{Ca}^{2+}$ measured as changes in single-cell $\mathrm{G} / \mathrm{R}$ ratios (Fig. $2 \mathrm{~b}, \mathrm{c}$ ). Moreover, depletion of Piezol by siRNA significantly inhibited Yodal-induced $\mathrm{Ca}^{2+}$ influx, confirming the specificity of Yoda1 in targeting Piezo1 while also validating reduced ion channel expression (Fig. 2d-e and Supplementary Video 1).

We next determined the role of Piezol in regulating $\mathrm{Ca}^{2+}$ events in response to cytokine-mediated macrophage activation. We first imaged Salsa6f + BMDMs as they were acutely exposed to Ringer solution-alone (control), and then containing IFN $\gamma / \mathrm{LPS}$ or IL4/IL13, allowing comparison of cytosolic $\mathrm{Ca}^{2+}$ responses between control and soluble stimuli conditions within the same field of view. Under these conditions, we observed both local and global elevations in cytosolic $\mathrm{Ca}^{2+}$ (Supplementary Fig. 8). More importantly, we found that stimulation with inflammatory cytokines (IFN $\gamma / \mathrm{LPS}$ ) led to greater $\mathrm{Ca}^{2+}$ activity, as indicated by increased number of $\mathrm{Ca}^{2+}$ events and percentage of cells actively showing $\mathrm{Ca}^{2+}$ influx (Supplementary Fig. 8, see methods section for details on quantification). No increases were observed with acute IL4/IL13 treatment, suggesting that increased $\mathrm{Ca}^{2+}$ activity may be a unique response to inflammatory stimuli (Supplementary Fig. 8). To determine the specific role of Piezo1 in this process, Salsa6f+ BMDMs were treated with control nontarget (siControl) or Piezol siRNA (siPiezol). siPiezol treatment prevented the increase in the number of $\mathrm{Ca}^{2+}$ events observed in response to acute addition of IFN $\gamma / \mathrm{LPS}$, which was maintained in siControl-treated BMDMs (Fig. 2f-h and Supplementary Video 2). These data establish a role for Piezol in mediating macrophage $\mathrm{Ca}^{2+}$ influx in response to acute inflammatory stimuli. In addition, we also examined changes in $\mathrm{Ca}^{2+}$ events in response to long-term exposure to inflammatory and healing activation stimuli. Imaging at a millisecond-scale temporal resolution using total internal reflection fluorescence (TIRF) microscopy ${ }^{17,47}$ revealed significantly higher $\mathrm{Ca}^{2+}$ activity at the membrane in inflammatory macrophages (Fig. 2i-k and Supplementary Video 3). These TIRF data show that local and transient $\mathrm{Ca}^{2+}$ events that lasted milliseconds in scale, which fall below the resolution of confocal microscopy, are prominent in inflammatory activated macrophages. Together, these results establish a role for Piezol in mediating $\mathrm{Ca}^{2+}$ signals in response to soluble inflammatory stimuli.

Stiffness-mediated macrophage activation is dependent on Piezo1. Substrate rigidity has been shown by our group and others to influence macrophage inflammatory activation in response to soluble stimuli ${ }^{5,9,10}$. Given that the Piezol channel is involved in mechanosensation in many cell types and its activity has previously been shown to be modulated by substrate stiffness ${ }^{17,18}$, we next investigated the role of stiffness in influencing $\mathrm{Ca}^{2+}$ and Piezol-mediated macrophage activation. Using fibronectin-conjugated polyacrylamide substrates of varying stiffness $(1,20,40$, and $280 \mathrm{kPa})$, we first evaluated the role of substrate stiffness on the expression of iNOS and ARG1 following IFN $\gamma /$ LPS and IL4/IL13 treatment, respectively. We found that increases in substrate stiffness are associated with enhanced iNOS expression in response to IFN $\gamma / \mathrm{LPS}$ stimulation, with significant increases in cells on $280 \mathrm{kPa}$ compared to 20 or $40 \mathrm{kPa}$ (Fig. 3a). These data are consistent with earlier reports ${ }^{5,9,10}$. In addition, we found that macrophages generally have increased IL4/IL13induced ARG1 expression when the stiffness of the substrate increases, with a significant difference between cells on 40 and $280 \mathrm{kPa}$ (Fig. 3a). Consistent with these findings, we found that stiff substrates enhance IFN $\gamma /$ LPS-induced NFKB and IL4/IL13induced STAT6 activation (Fig. 3b-c). Inhibition of STAT6 using AS1517499 suppressed Arg1 expression in response to IL4/ IL13 stimulation in cells seeded on both 1 and $280 \mathrm{kPa}$ surfaces, suggesting that STAT6 mediated healing activation is present even on soft substrates (Supplementary Fig. 9). Together, these data support the involvement of NFKB and STAT6 in stiffnessdependent macrophage activity.

We next evaluated whether Piezol contributes to the observed effects on inflammatory and healing activation of BMDMs cultured on different stiffness substrates. To assess Piezo1 expression, we utilized BMDMs from Piezo1 ${ }^{P 1-t d T}$ mice which express a Piezo1-tdTomato fusion protein that labels endogenous Piezol channels with tdTomato ${ }^{21}$. We found that Piezo1 expression increased in macrophages cultured on stiff $(280 \mathrm{kPa})$ compared to soft $(1 \mathrm{kPa})$ substrates using both immunofluorescence imaging of tdTomato and Western blot of Piezol (Fig. 3d and Supplementary Fig. 10). We also examined $\mathrm{Ca}^{2+}$ influx in Salsa6f + BMDMs seeded on different stiffness substrates. BMDMs seeded on stiffer substrates displayed more frequent $\mathrm{Ca}^{2+}$ events with a larger percentage of cells actively involved in $\mathrm{Ca}^{2+}$ influx following exposure to IFN $\gamma / \mathrm{LPS}$ when compared to cells cultured on soft substrates, suggesting that stiffnessdependent inflammatory activation is associated with increased $\mathrm{Ca}^{2+}$ activity (Fig. 3e-f, Supplementary Fig. 10, and Supplementary Video 4). Consistent with these findings, Salsa6f + BMDMs seeded on substrates of higher stiffnesses showed significantly 
a

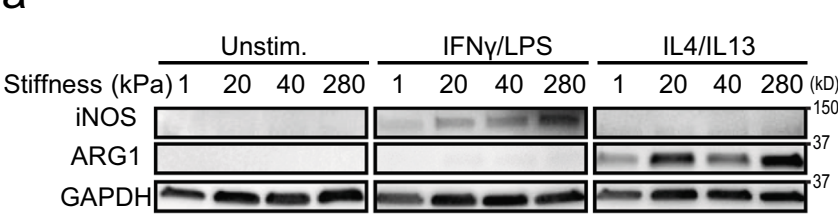
b $_{\text {Stiffness (kPa) } 1280} \frac{\text { Unstim. IFNy/LPS }}{1280(\mathrm{kD})} \mathrm{C}_{\text {Stiffness (kPa) } 1280} \frac{\text { Unstim. }}{\frac{\text { IL4/L13 }}{1280}(\mathrm{kD})}$

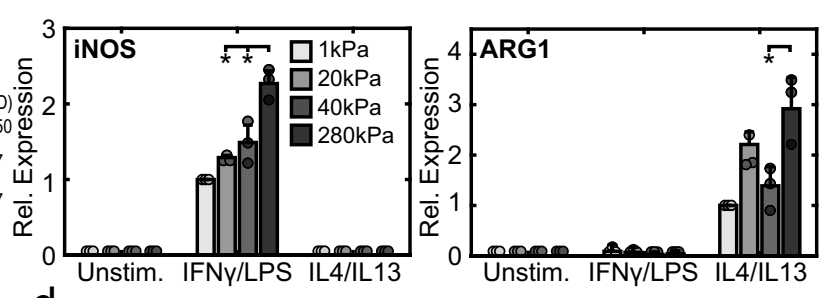
$\mathrm{p}-\mathrm{NFKB}$ $\mathrm{NFKB}$
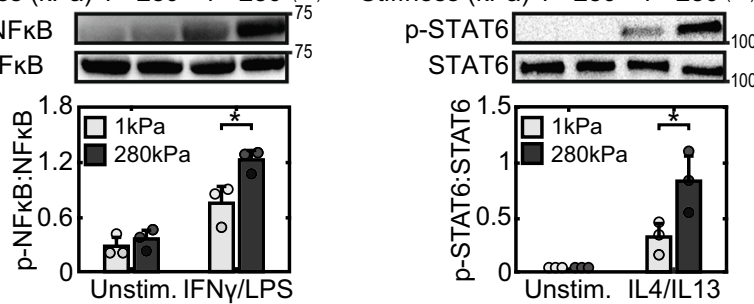

e
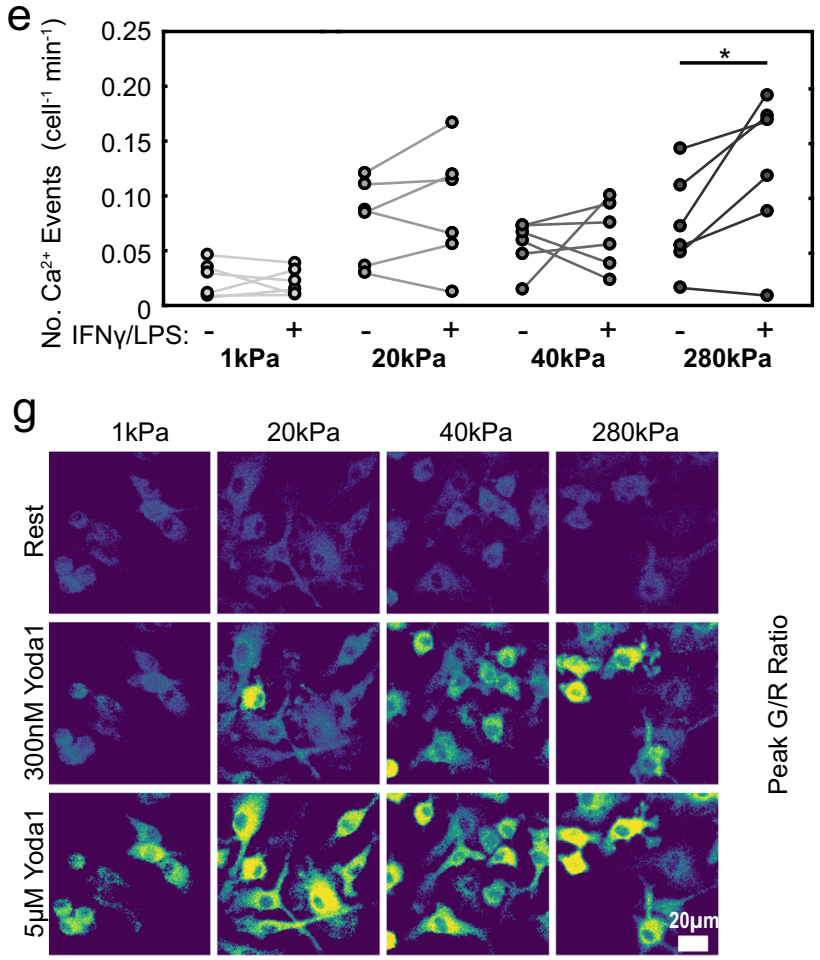

G/R Ratio: Low
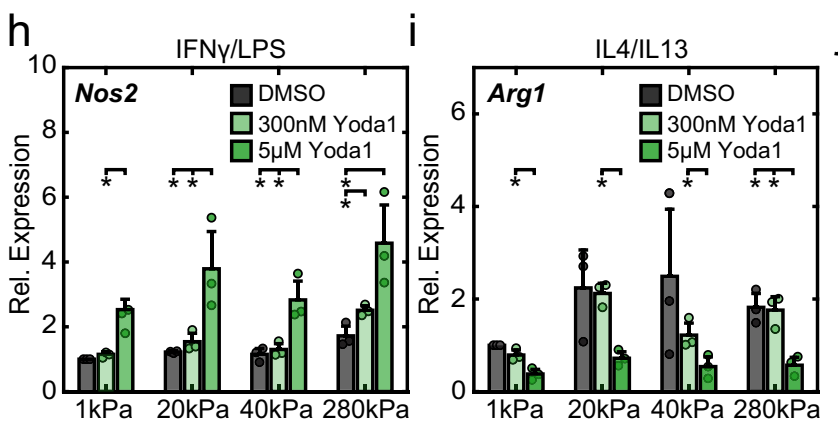

larger $\mathrm{Ca}^{2+}$ responses to Yodal $(300 \mathrm{nM})$ (Fig. 3g). These differences were not observed at higher doses of Yodal $(5 \mu \mathrm{M})$, likely due to a maximum global increase in cytosolic $\mathrm{Ca}^{2+}$ that obscures the differences in Piezol expression across different stiffnesses. Nonetheless, these results suggest that substrate
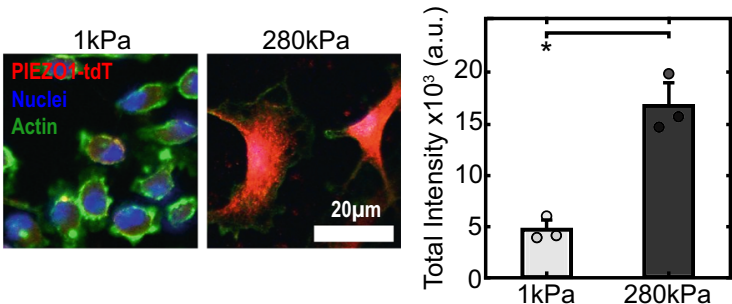

f
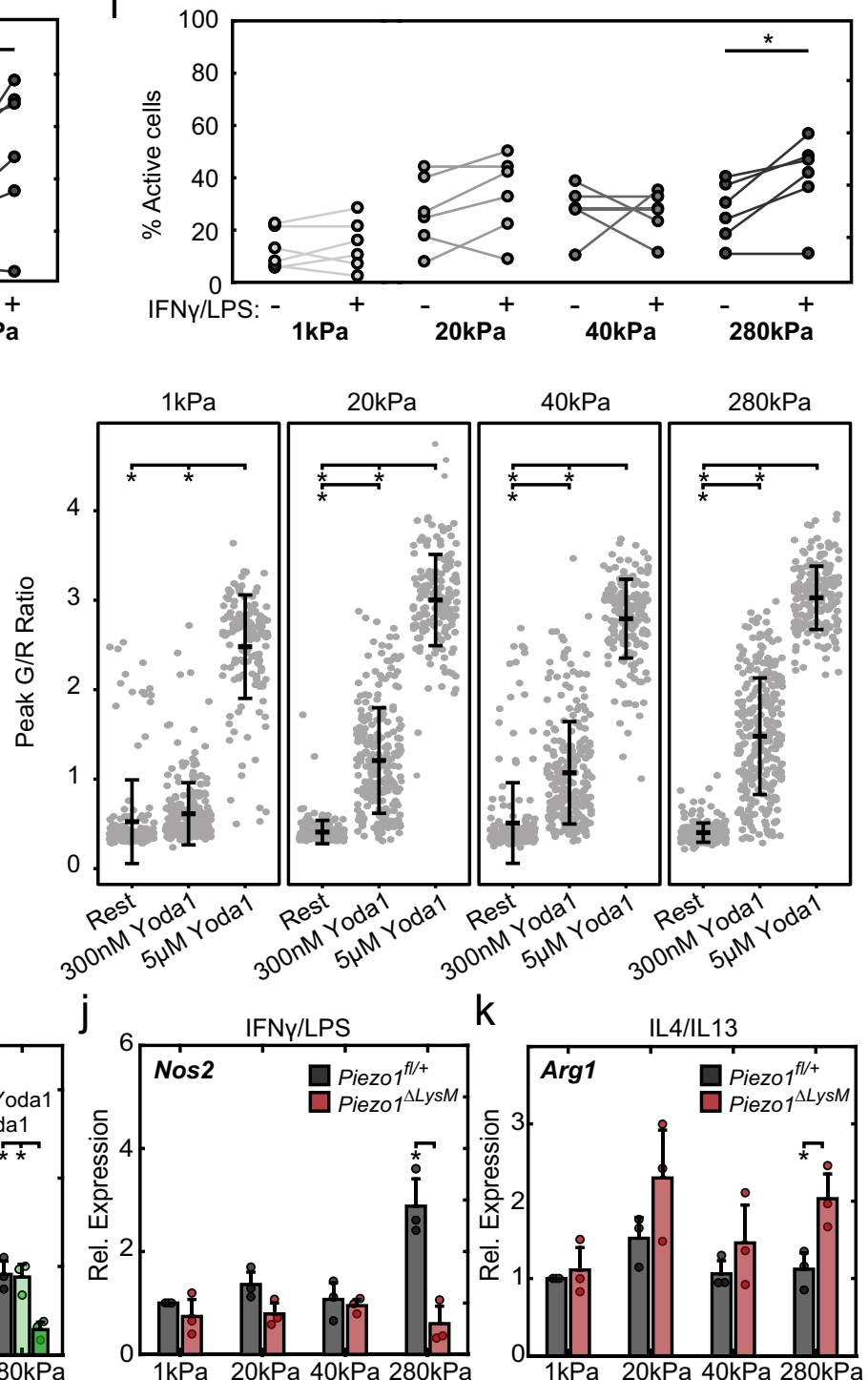

stiffness increases the expression of Piezol at the cell membrane (Fig. $3 \mathrm{~g}$ and Supplementary Video 5).

To probe the role of Piezol in stiffness-dependent changes in macrophage function, we first used Yodal on wild type BMDMs seeded on different stiffness substrates. We found that low doses 
Fig. 3 Stiffness-dependent Piezo1 expression/activity modulates macrophage activation. a Representative Western blots (left) and quantification (right) of iNOS, ARG1, and GAPDH of BMDMs seeded on 1, 20, 40, and $280 \mathrm{kPa}$ polyacrylamide gels and incubated with media (Unstim.), $0.3 \mathrm{ng} / \mathrm{mL}$ IFN $\gamma / \mathrm{LPS}$, or $0.1 \mathrm{ng} / \mathrm{mL}$ IL4/IL13. Data normalized to the $1 \mathrm{kPa}$ IFN $/$ /LPS condition. Samples were derived from the same experiment and the resulting blots were processed in parallel. b, c Representative Western blots (top) and quantification (bottom) of p-NFkB/NFkB (b) and p-STAT6/STAT6 (c) in wild type BMDMs seeded on 1 and $280 \mathrm{kPa}$ polyacrylamide gels and stimulated with IFN $\gamma /$ LPS or IL4/IL13, respectively. Phosphorylated and total forms of transcription factors were obtained from loading equal amounts of protein in separate gels and the resulting blots were processed in parallel. $\mathbf{d}$ Representative immunofluorescent images (left) and quantification of fluorescence (right) of Piezo ${ }^{\text {P1-tdT }}$ BMDMs seeded on polyacrylamide gels, tdTomato is displayed in red. Quantification of $\mathbf{e}$ number of $\mathrm{Ca}^{2+}$ events (normalized for cell number and time) and $\mathbf{f}$ fraction of cells showing $\mathrm{Ca}^{2+}$ elevations in unstimulated BMDMs seeded on 1, 20, 40, and $280 \mathrm{kPa}$ polyacrylamide surfaces following acute addition of Ringer solution (Unstim.) or Ringer solution with $100 \mathrm{ng} / \mathrm{mL}$ IFN $\gamma /$ LPS, captured by confocal microscopy. Each data point is calculated from a 10-min time-lapse video ( $N=6$ for each stiffness). $\mathbf{g}$ Representative G/R ratio images (left) and quantification of peak G/R intensities of BMDMs seeded on polyacrylamide gels of indicated stiffness and exposed to DMSO, $300 \mathrm{nM}$ Yoda1, and $5 \mu \mathrm{M}$ Yoda1. $N=107,248,145$ cells on $1 \mathrm{kPa}, n=118,239$, 169 cells on $20 \mathrm{kPa}, n=113,250,183 \mathrm{cells}$ on $40 \mathrm{kPa}$, and $n=106,274,184$ cells on $280 \mathrm{~Pa}$ and exposed to Rest, $300 \mathrm{nM}$ Yoda1, or $5 \mu \mathrm{M}$ Yoda1, respectively. Data representative of three independent experiments. h Relative Nos2 gene expression of BMDMs seeded on surfaces of indicated stiffness, exposed to DMSO, $300 \mathrm{nM}$ Yoda1, or $5 \mu \mathrm{M}$ Yoda1, and stimulated with $0.3 \mathrm{ng} / \mathrm{mL}$ IFN $\gamma /$ LPS. Data normalized to $1 \mathrm{kPa}$ DMSO control. i Relative Arg1 gene expression of BMDMs seeded on surfaces of indicated stiffness, exposed to DMSO, $300 \mathrm{nM}$ Yoda1, or $5 \mu \mathrm{M}$ Yoda1, and stimulated with $0.1 \mathrm{ng} / \mathrm{mL}$ IL4/IL13. Data normalized to $1 \mathrm{kPa}$ DMSO control. $\mathbf{j}$ Relative Nos2 gene expression Piezo $7^{f / /}+$ and Piezo ${ }^{\Delta L y s M}$ BMDMs seeded on surfaces of indicated stiffness and stimulated with $0.3 \mathrm{ng} / \mathrm{mL}$ IFN $\gamma / \mathrm{LPS}$. Data

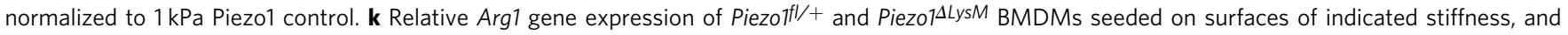
stimulated with $0.1 \mathrm{ng} / \mathrm{mL}$ IL4/IL13. Data normalized to $1 \mathrm{kPa}$ Piezo $7 \mathrm{fl} /+$ controls. Error bars denote Mean \pm SD for three separate experiments, ${ }^{\star} p<0.05$ as determined by two-tailed paired $t$ test $(\mathbf{a}, \mathbf{e}, \mathbf{h}-\mathbf{k})$, two-tailed Student's test $(\mathbf{b}-\mathbf{d})$, or Mann-Whitney $U$ test $(\mathbf{g})$. Source data including exact $\mathrm{p}$-values are provided as a Source Data file.

of Yoda1 (300 nM) significantly increased the gene expression of the inflammatory marker Nos 2 in IFN $\gamma /$ LPS-stimulated BMDMs cultured on $280 \mathrm{kPa}$ hydrogels. High doses of Yodal $(5 \mu \mathrm{M})$ significantly enhanced Nos2 expression across all stiffness substrates (Fig. 3h). High doses of Yodal also significantly decreased the expression of the healing marker Arg1 in IL4/IL13stimulated cells (Fig. 3i). In contrast to Yoda1-induced channel activation, Piezo1 $1^{\triangle L y s M}$ BMDMs cultured on stiff substrates exhibited less Nos 2 in response to IFN $\gamma /$ LPS and increased Arg1 expression in response to IL4/IL13 (Fig. 3j-k). Taken together, these results support a model whereby stiffer environments are associated with increased Piezol expression and $\mathrm{Ca}^{2+}$ activity, which enhances inflammation. Using a pharmacological approach, we confirmed the role of calpains, $\mathrm{Ca}^{2+}$ dependent proteases, in regulating IFN $\gamma /$ LPS induced inflammatory activation in macrophages, as has previously been shown by others ${ }^{48,49}$ (Supplementary Fig. 11). Intriguingly, while increases in stiffness were associated with increased IL4/IL13-induced wound healing response, enhanced activation of Piezol by Yodal inhibited wound healing and inhibition of Piezol by genetic knockout enhanced healing, suggesting that additional mechanisms independent of Piezol are likely involved in stiffness-mediated enhancement of healing responses. It is possible that other ion channels may be involved, since broad inhibition of ion channels by $\mathrm{GdCl}_{3}$ or $\mathrm{GsMTx}-4$ resulted in reduced expression of healing markers in response to IL4/IL13 (Supplementary Figs. 6, 7). Nonetheless, these results suggest a critical role for stiffness in regulating $\mathrm{Ca}^{2+}$ activity in macrophages and show that these effects, particularly in the context of inflammation, are dependent on Piezol.

Macrophage responses to different stiffness material implants in-vivo is regulated by Piezo1. Mechanical mismatch is common between surgical implants and native tissues, and often results in poor material integration characterized by a severe foreign body response $^{11}$. While stiff materials are often favored due to their mechanical integrity, they have also been shown to promote inflammation and are associated with thicker fibrous collagen capsule formation when compared to soft materials ${ }^{11,12}$. To better understand the potential role of Piezol in regulating the response of myeloid cells, including macrophages, during this process, we implanted precast soft $(1 \mathrm{kPa})$ and stiff $(140 \mathrm{kPa})$ polyethylene glycol diacrylate-400 (PEGDA-400) hydrogels subcutaneously into Piezo ${ }^{f l /+}$ and Piezo1 ${ }^{\Delta L y s M}$ mice. After 14 days of implantation, the hydrogels and surrounding tissue were harvested and examined by histology to quantify immune cell infiltration and fibrous capsule thickness (Fig. $4 a-d$ ). In control Piezol ${ }^{f l /}$ mice, we found that stiff material implants significantly increased immune cell infiltration and caused a more severe foreign body response, as indicated by thicker fibrous capsule formation, when compared to soft material implants, consistent with previous reports in wild type mice ${ }^{10,12}$. In contrast, depletion of Piezo1 in myeloid cells abrogated these responses to stiff implants with significantly reduced immune infiltrate and thickness of the collagen capsule surrounding the implant material, comparable to soft implants. These data suggest that the expression of Piezol in cells of myeloid lineage plays a pivotal role in influencing the host response to different stiffness material implants.

Given that myeloid-derived macrophages are central regulators of the foreign body response, we next examined the expression of macrophage functional markers in response to implants. Tissues surrounding soft and stiff implants in Piezo1 ${ }^{f l /+}$ and Piezo1 ${ }^{\Delta L y s M}$ mice were harvested at 3 and $14 \mathrm{~d}$ after implantation, and immunohistochemistry was used to evaluate macrophage specific (F4/80+) expression of iNOS and ARG1 (Fig. 4e-f). We observed that the extent of macrophage recruitment to soft and stiff implants was similar between Piezol $1^{f l+}$ and Piezo $1^{\Delta L y s M}$ mice at both 3 and $14 \mathrm{~d}$ after implantation (Fig. $4 \mathrm{~g}$ ). However, there were significant differences in expression of functional markers. In control Piezo $1^{f l /+}$ mice, the percentage of iNOS + macrophages surrounding stiff implants was greater at both 3 and $14 \mathrm{~d}$ (Fig. 4h-i), consistent with in vitro observations (Fig. 3a). However, unlike the in vitro findings, the percentage of ARG1+ macrophages was reduced in stiff implants when compared to soft implants at $14 \mathrm{~d}$ in control Piezo $1^{f l /}+$ mice. These differences could potentially be attributed to the time point of evaluation. In Piezo1 ${ }^{\triangle L y s M}$ mice, the percentage of iNOS + macrophages was similar and low in response to soft and stiff materials; the percentage of ARG1+ macrophages was also similar in response to soft and stiff materials at $3 \mathrm{~d}$, and increased at $14 \mathrm{~d}$ (Fig. $4 \mathrm{~h}-\mathrm{i}$ ). We also found significant increases in the percentage of NFkB+ macrophages within tissue surrounding stiff compared to soft implants in Piezo1 ${ }^{f l+}$ mice, but not Piezo1 ${ }^{\Delta L y s M}$ mice which is consistent with our observations of reduced inflammation in 

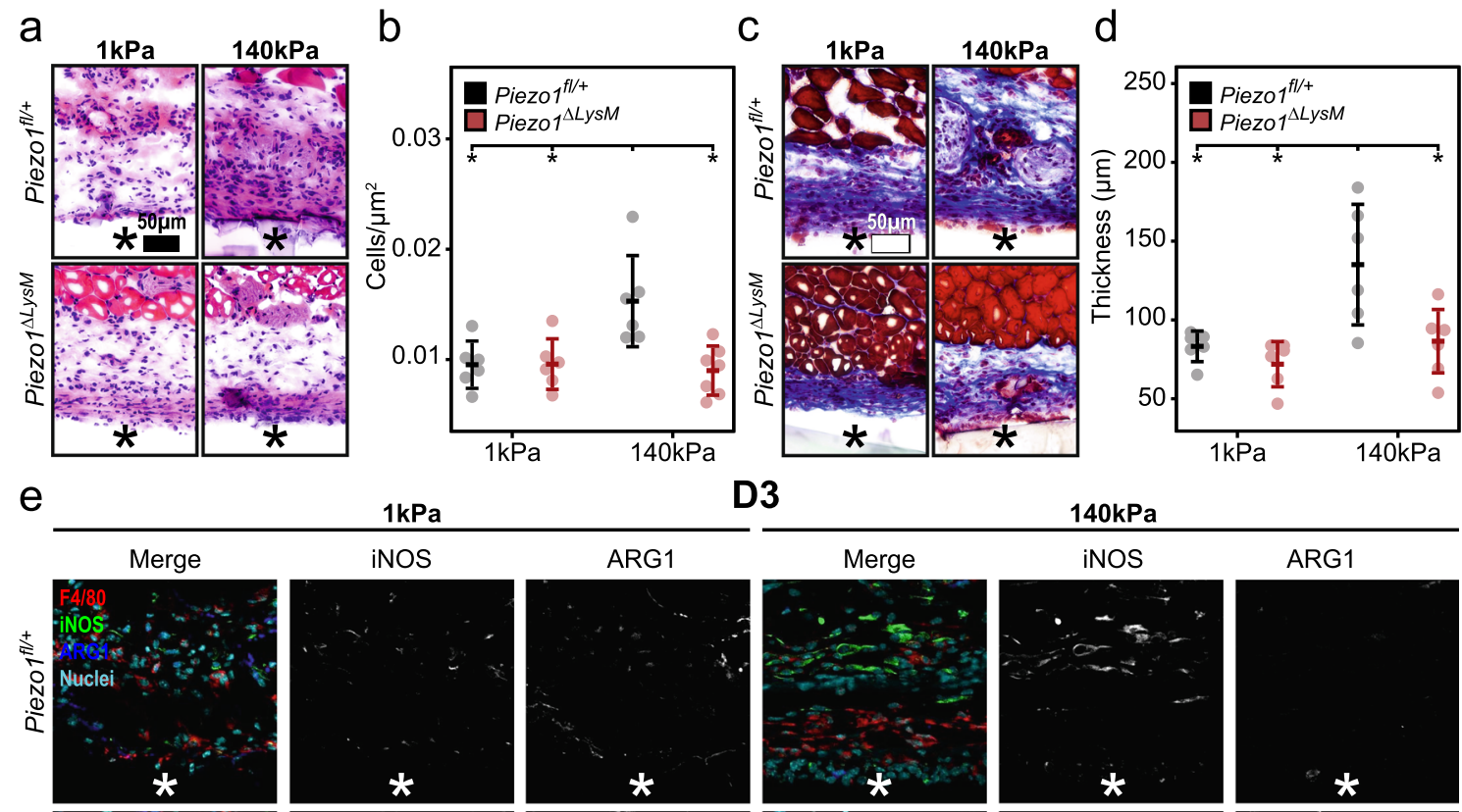

\begin{abstract}
$1 \mathrm{kPa}$
\end{abstract}
D3
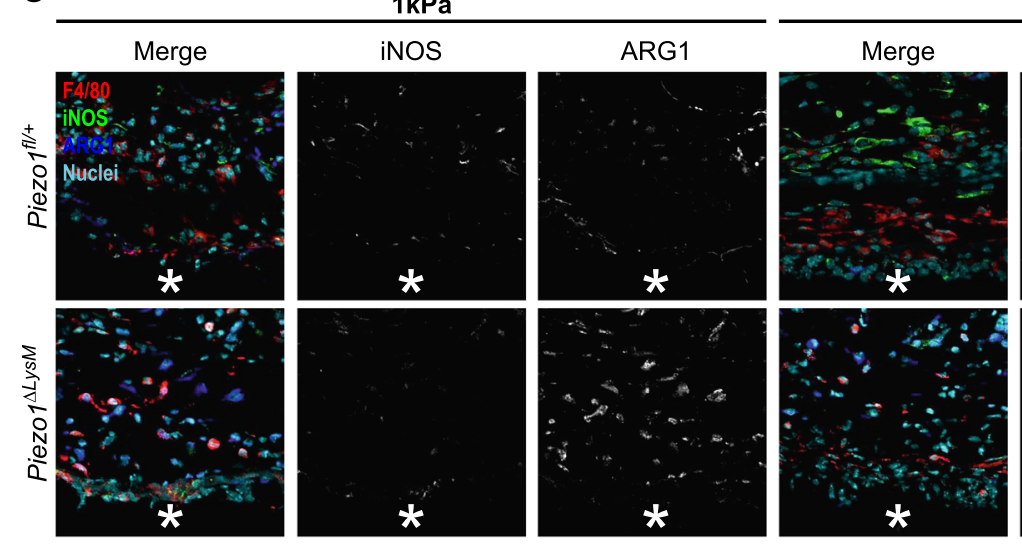

$140 \mathrm{kPa}$

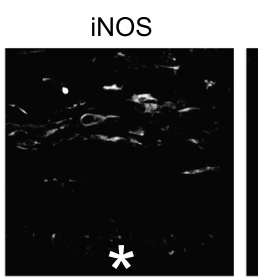

ARG1
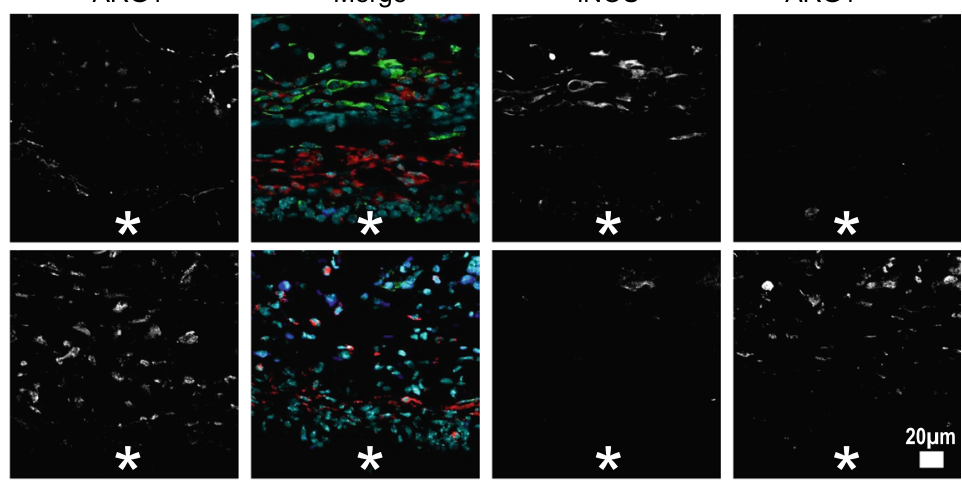

$f$

$1 \mathrm{kPa}$

D14
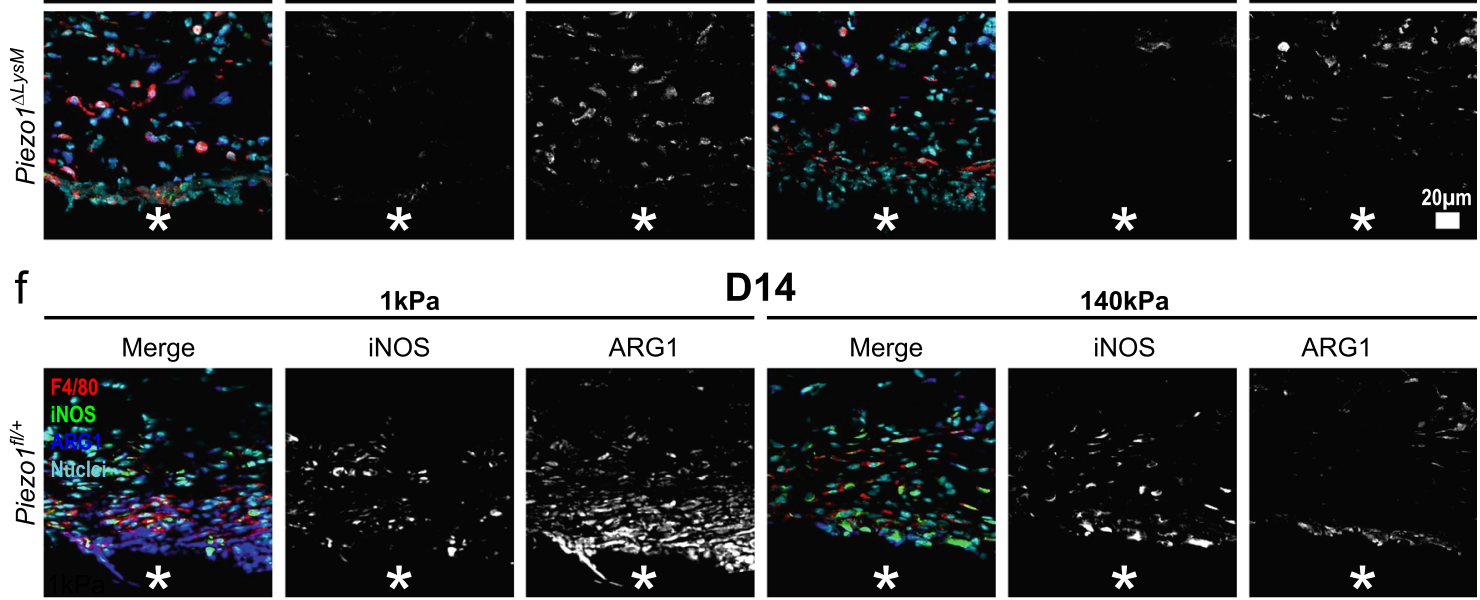

Merge
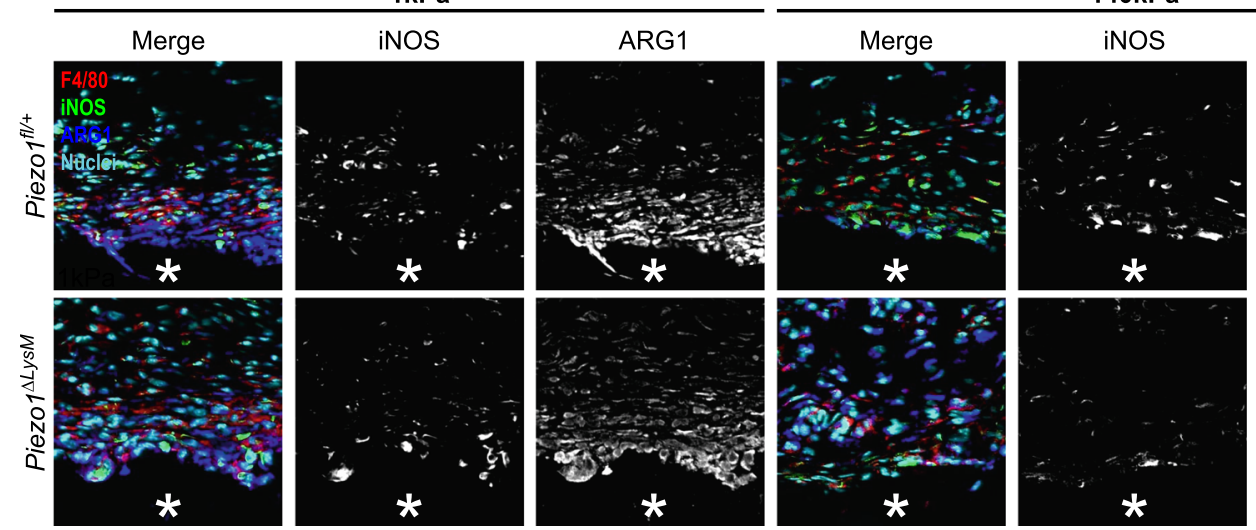

ARG1
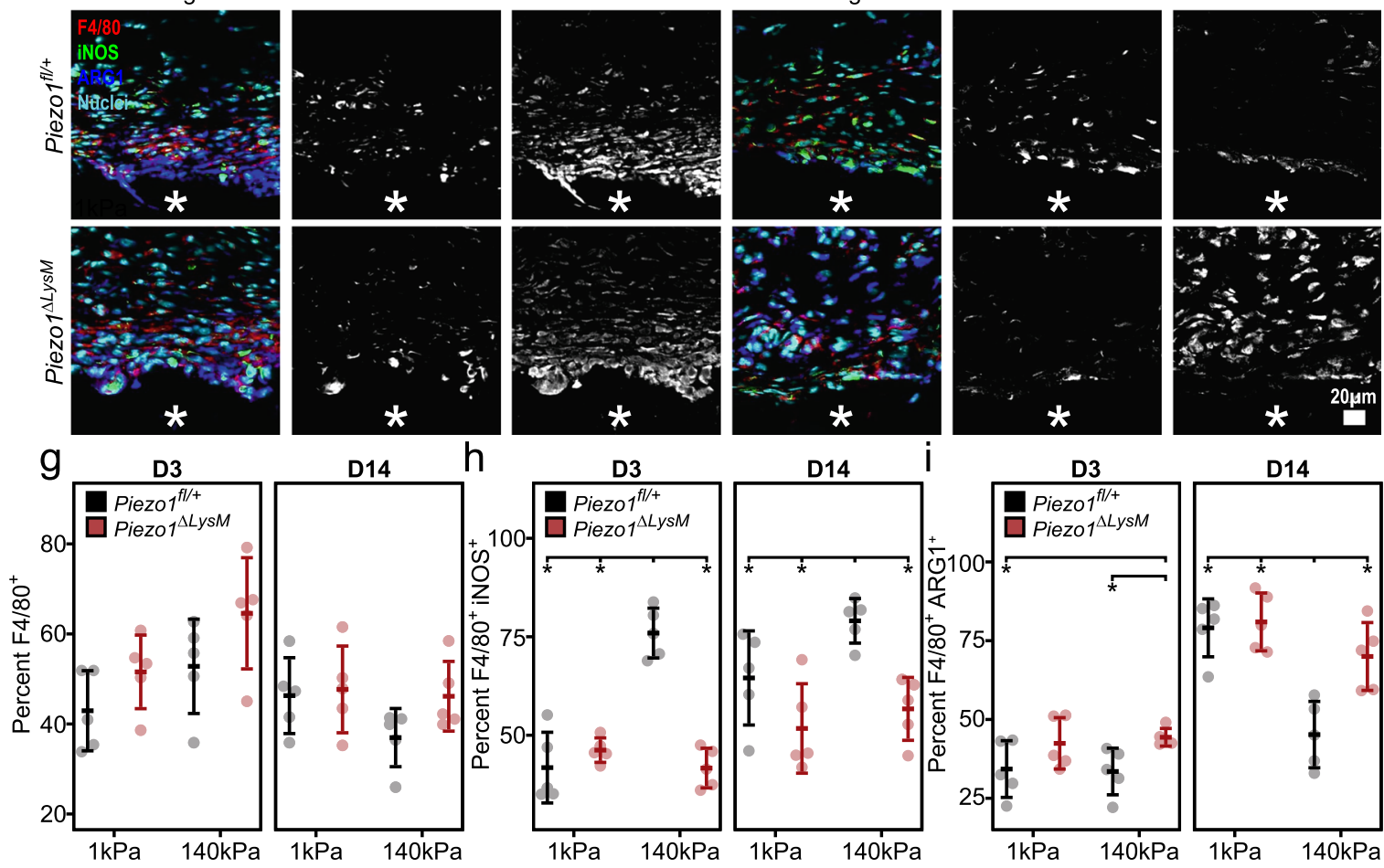

myeloid cell-specific Piezol deficient mice (Supplementary Fig. 12). These data again suggest that macrophages lacking Piezol may lose their ability to sense and respond to different stiffness environments. The decrease in inflammatory activation and increase in healing activation support the reduced immune infiltrate and fibrous capsule thickness observed in Piezo1 $1^{\Delta L y s M}$ mice. Together, these results further validate the role of Piezol in regulating macrophage polarization responses to different stiffness environments and reveal downstream effects on fibrous capsule formation. 
Fig. 4 Piezo1 modulates the foreign body response and macrophage activation in response to stiff material implants. a-d Representative H\&E (a) and Masson's trichrome (c) stained tissue surrounding soft $(1 \mathrm{kDa})$ and stiff $(140 \mathrm{kDa})$ PEGDA material implanted in Piezo $\mathrm{fl}^{\mathrm{f} / \mathrm{+}}$ and Piezo ${ }^{\mathrm{ALysM}}$ mice for a period of 14 days. Quantification of immune cell infiltrate normalized to area $(\mathbf{b})$ and average collagen capsule thickness $(\mathbf{d})$. $N=6$ for Piezo $f^{f / /}+$ mice treated with soft and stiff implants as well as Piezo ${ }^{\Delta L y s M}$ treated with soft implants, and $n=7$ for Piezo $f^{f / /}+$ and Piezo ${ }^{\Delta L y s M}$ mice treated with stiff implants. e, $\mathbf{f}$ Representative immunohistochemistry images of tissue collected 3 days (D3, e) and 14 days (D14, f) post-implantation and stained for F4/80, iNOS, ARG1, and Hoechst. $\mathbf{g}$ Quantification of percent cells that stained positive for F4/80. $\mathbf{h}$ Quantification of percent cells that stained positive for F4/80 and iNOS. i Quantification of percent cells that stained positive for F4/80 and ARG1. Material location indicated with asterisk. $N=5$ and error bars denote Mean \pm SD for $n \geq 5,{ }^{\star} p<0.05$ as determined by two-tailed Student's $t$ test. Source data including exact $p$-values are provided as a Source Data file.

Cytoskeletal feedback regulates Piezo1-mediated $\mathrm{Ca}^{2+}$ influx and macrophage activation. While Piezol is well known for its ability to transduce external mechanical stimuli ${ }^{22-24}$, this channel has also been shown to be activated by internal cell-generated forces, which are largely governed by the cytoskeleton ${ }^{17,47,50}$. In addition, changes in the actin cytoskeleton have been observed in response to manipulation of Piezo1, thus suggesting a potential coregulation of the cytoskeleton and ion channel ${ }^{18,23,50,51}$. Given recent reports highlighting the importance of actin in the modulation of macrophage activation ${ }^{52,53}$, we next sought to better understand the interplay between actin and Piezol, and their role in regulating inflammation. We first evaluated changes in actin resulting from Piezol modulation by staining with phalloidin, which binds to F-actin. We found that Yoda1-mediated channel activation resulted in significantly enhanced F-actin mean intensity compared to DMSO controls (Fig. 5a). Conversely, Piezo $1^{\Delta L y s M}$ macrophages were observed to have significantly reduced F-actin intensity when compared to Piezo ${ }^{f l /+}$ controls (Fig. 5b). We also evaluated the effect of substrate rigidity and found that cells cultured on stiffer surfaces $(20,40,280 \mathrm{kPa})$, which were previously observed to have increased Piezol expression, exhibited enhanced F-actin levels-integrated across the cell area, which also changes with stiffness-compared to cells on soft $1 \mathrm{kPa}$ surfaces (Supplementary Fig. 13). These results suggest that Piezol activity enhances actin polymerization in macrophages.

We next sought to examine the role of actin in regulating Piezo1-mediated $\mathrm{Ca}^{2+}$ influx and macrophage activation. We first exposed BMDMs to latrunculinA (LatA), a potent actin inhibitor, and jasplakinolide (Jasp), an actin stabilizer, and quantified changes in F-actin intensity. As expected, we found that LatA reduced and Jasp enhanced F-actin intensity when compared to DMSO controls (Fig. 5c). We next exposed non-targeting (siControl) and Piezol (siPiezo1) siRNA treated Salsa6f + BMDMs to DMSO, LatA, or Jasp and evaluated for changes in IFN $\gamma /$ LPS-induced $\mathrm{Ca}^{2+}$ activity. Consistent with our previous results, we observed a reduction in $\mathrm{Ca}^{2+}$ activity in siPiezol when compared to siControl treated cells in the DMSO condition (Fig. $5 \mathrm{~d}-\mathrm{g}$ ). We also found that cells exposed to LatA resulted in reduced $\mathrm{Ca}^{2+}$ activity in both siControl and siPiezol treated BMDMs. In contrast, treatment with Jasp enhanced $\mathrm{Ca}^{2+}$ activity in siControl treated cells, which was reduced by siPiezol treatment, although the levels were higher than DMSO and LatA treated siPiezol cells. These data suggest that inhibition of actin polymerization with LatA reduces IFN $\gamma / \mathrm{LPS}$ induced $\mathrm{Ca}^{2+}$ activity, whereas stabilization of actin with Jasp promotes Piezo1 activity and may additionally stimulate other $\mathrm{Ca}^{2+}$ channel activity (Fig. 5d-g). We also found that pharmacological inhibition of myosin II using ML7 inhibited Piezo1-mediated $\mathrm{Ca}^{2+}$ influx (Supplementary Fig. 14). Together these data suggest a positive feedback regulation, with Piezol activity enhancing actin polymerization, which in turn increases Piezol activity.

We next examined the role of actin and Piezol in modulating inflammatory activation by treating cells with LatA or Jasp and evaluating macrophage response to inflammatory agonists.
Piezo1 ${ }^{\triangle L y s M}$ macrophages treated with DMSO generally had reduced IFN $\gamma /$ LPS mediated inflammatory gene expression when compared to Piezo1 ${ }^{f l+}$ DMSO controls, consistent with our earlier results (Fig. $5 \mathrm{~h}-\mathrm{j}$ ). We also observed that cells treated with LatA had suppressed inflammatory activation in Piezol $\mathrm{fl} /+$ macrophages, which was similar to the behavior of Piezo1 ${ }^{\Delta L y s M}$ macrophages treated with DMSO, and further knock out of Piezol had no effect (Fig. 5h). In contrast, Piezo $1^{\Delta L y s M}$ and Piezo1 ${ }^{f l /}+$ BMDMs treated with Jasp had enhanced expression of the inflammatory genes, Il6 and Il1 b, when compared to DMSO controls. Expression of Mcp1 was reduced, similar to what was previously observed with Yodal treatment (Fig. $5 \mathrm{~h}-\mathrm{j}$ ). Together, these studies confirm the role of actin in regulating macrophage activation, as has previously been observed ${ }^{52,53}$, and also suggest a potential for positive feedback between Piezol and the actin cytoskeleton which, in turn, enhances macrophage inflammatory activation.

\section{Discussion}

Macrophages encounter environments of varied stiffnesses as they are recruited to tissues throughout the body to facilitate inflammatory and healing responses after injury or infection. In this study, we identify a role for the mechanically activated ion channel Piezol in macrophage stiffness sensing, and their responses to inflammatory and wound healing agonists. We find that Piezol is highly expressed in macrophages derived from bone marrow, and its activity enhances IFN $\gamma / \mathrm{LPS}$ and suppresses IL4/IL13-induced activation through increased $\mathrm{NF \kappa B}$ and decreased STAT6 activation, respectively (Fig. 6). Opposing regulation of inflammatory and wound healing states have also been reported for other transcription factors, KLF4 and KLF6, as well as signaling molecules, AKT1 and AKT2 ${ }^{54-56}$, which upregulate the expression of inflammatory molecules that simultaneously suppress healing activation, such as $\mathrm{TNFa}^{54,56,57}$. While our data suggest that manipulation of Piezol itself has no effect on unstimulated macrophages, activation or deletion of this ion channel may indeed regulate molecules that are capable of switching macrophage phenotypes. We also show that Piezol regulates the activation of resident peritoneal macrophages. While continuous mechanical stimuli present in vivo are thought to desensitize Piezol in resident alveolar macrophages ${ }^{27}$, the lung and peritoneum clearly have distinct mechanical environments, which may differentially modulate Piezol activity. In addition, culture of cells in vitro may potentially cause loss of mechanical memory in resident macrophage populations and mask the effects of desensitization. Nevertheless, our studies suggest a major role for Piezol in modulating the functional responses of broad macrophage populations.

$\mathrm{Ca}^{2+}$ plays a pivotal role in regulating essential macrophage functions including inflammatory activation ${ }^{25,26}$. Using Salsa6fexpressing macrophages, we show $\mathrm{Ca}^{2+}$ influx in response to the chemical Piezo1 agonist Yoda1, IFN $\gamma /$ LPS stimulation, and to an extent IL4/IL13 stimulation. While the response of Piezo1 to chemical agonist Yodal has been known for some time ${ }^{44}$, the relevance of chemical activation of Piezol under physiological 
a
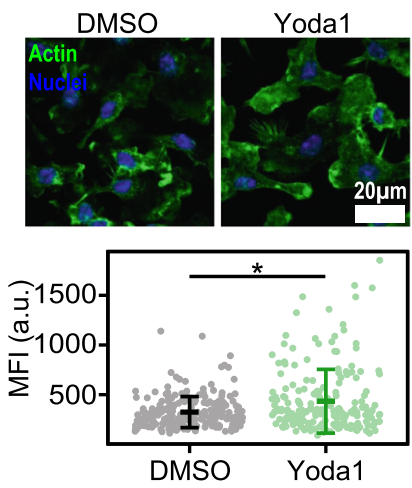

d
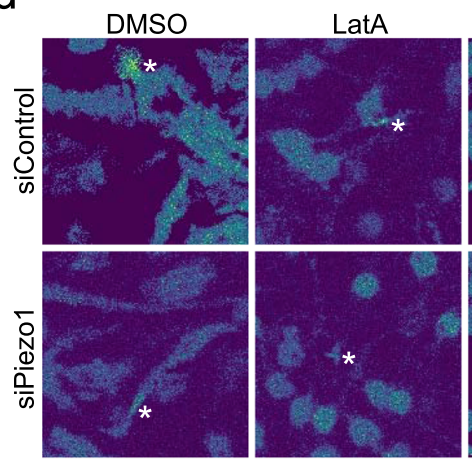

G/R Ratio:

f

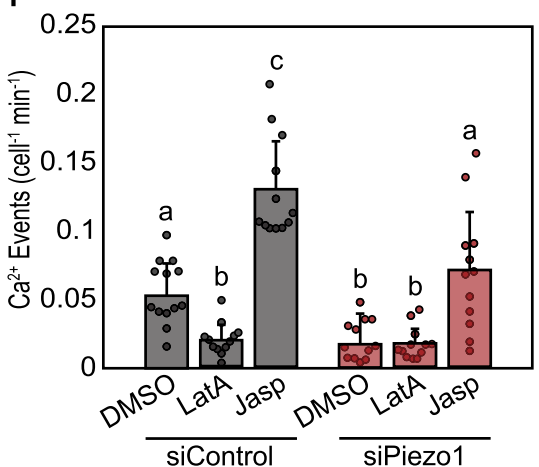

b
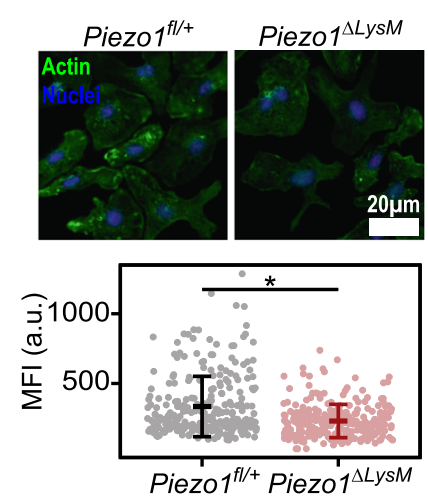

e
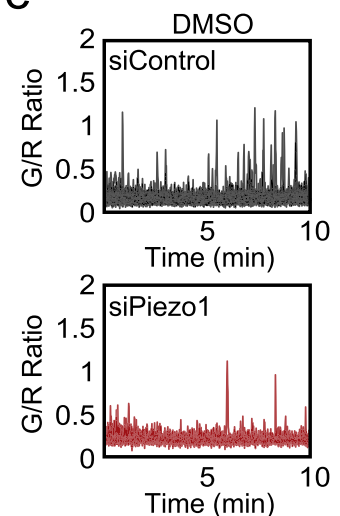

C
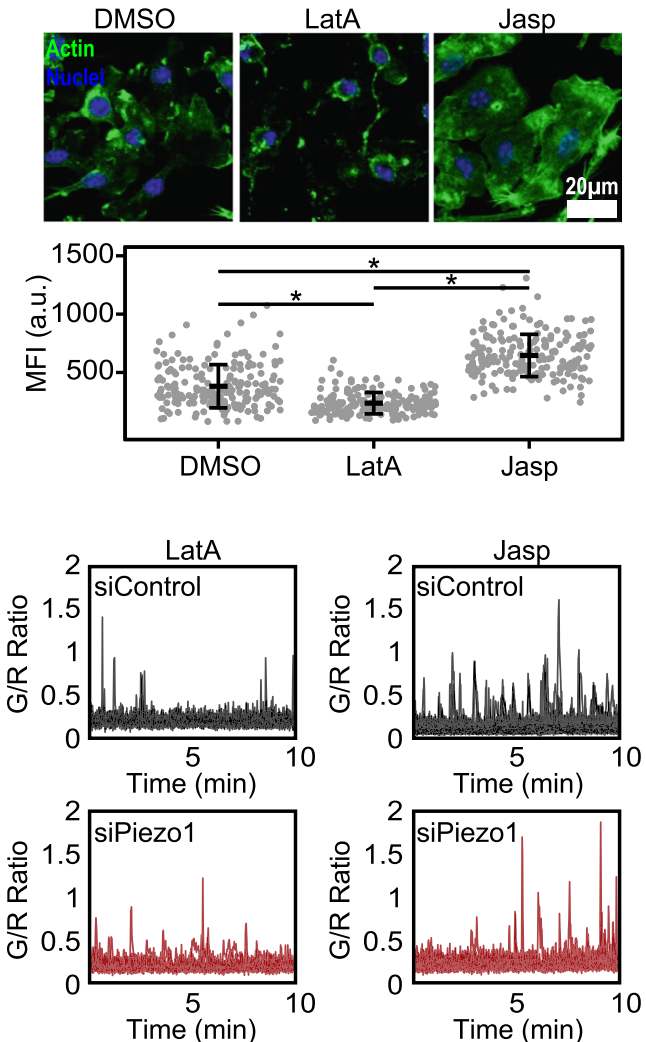

h $g$

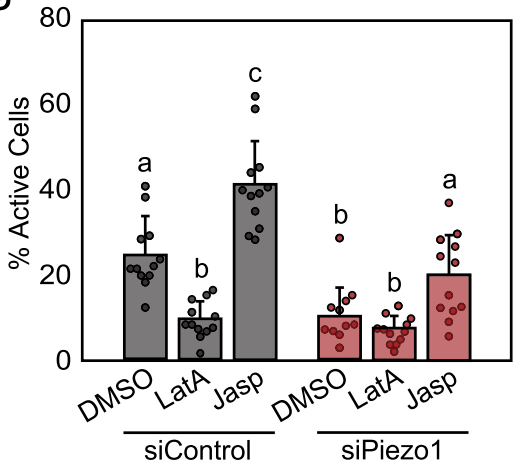

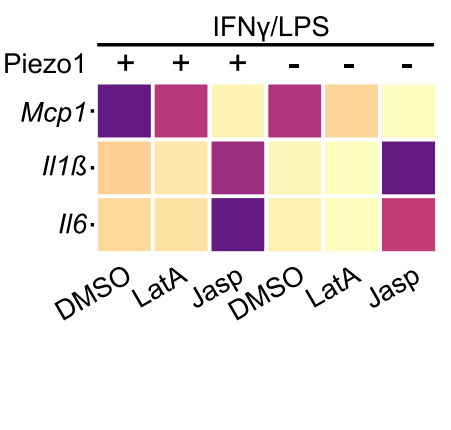

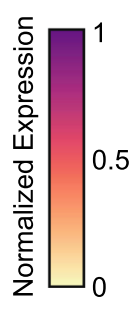

Fig. 5 Piezo1-mediated regulation of actin influences macrophage inflammatory activation. a Representative images (top) and quantification (bottom) of actin mean fluorescent intensity (MFI) of wild-type BMDMs following $1 \mathrm{~h}$ treatment with DMSO or Yoda1. N=229 and 201 cells examined over three independent experiments for DMSO and Yoda1 treatment. b Representative images (top) and quantification (bottom) of actin MFI of Piezo fl/ + and Piezo $7^{\Delta L y s M}$ BMDMs. $N=277$ and 279 cells examined over three independent experiments for Piezo $7 f /+$ and Piezo $7^{\Delta L y s M}$ BMDMs. c Representative images (top) and quantification (bottom) of actin MFI in BMDMs following one-hour treatment with DMSO, $500 \mathrm{nM}$ latrunculinA (LatA), or $500 \mathrm{nM}$ jasplakinolide (Jasp). $N=195,177$, and 191 cells examined over three independent experiments for DMSO, LatA, and Jasp treatment. d-g Representative $\mathrm{G} / \mathrm{R}$ ratio images (d), traces of individual $\mathrm{Ca}^{2+}$ events (e), and quantification of number of $\mathrm{Ca}^{2+}$ events (normalized for cell number and time) and fraction of cells showing $\mathrm{Ca}^{2+}$ elevations, $(\mathbf{f}, \mathbf{g})$ taken from a time-lapse video of siControl and siPiezo1 treated Salsa6f+ BMDMs following addition of DMSO, LatA, or Jasp in Ringer solution containing $100 \mathrm{ng} / \mathrm{mL}$ IFN $\gamma /$ LPS. Asterisks denote the occurrence of a $\mathrm{Ca}^{2+}$ event. Each data point in (f, $\left.\mathbf{g}\right)$ denotes a single video $\left(N=12\right.$ videos). h Relative $\|6\|$,$17 b , and Mcp1 gene expression in Piezo f^{f l /+}$ and Piezo ${ }^{\Delta L y s M}$ BMDMs exposed to DMSO, LatA, or Jasp and stimulated with IFN $\gamma /$ LPS for 6 hrs. Gene expression is shown relative to the highest expressing condition. For (a-c), error bars denote Mean \pm SD, ${ }^{\star} p<0.05$ as determined by two-tailed Mann-Whitney $U$ test. For $(\mathbf{f}, \mathbf{g})$, error bars denote Mean $\pm S D$ for $n=3$, groups not connected by the same letter are statistically different $(p<0.05)$ as determined by two-tailed Student's $t$ test. Source data including exact $p$-values are provided as a Source Data file.

conditions remains unknown. We propose that soluble cues, such as IFN $\gamma / \mathrm{LPS}$, may prime the channel's response to mechanical cues, thus regulating the downstream responses to mechanical signals in different contexts. It is possible that $\mathrm{Ca}^{2+}$ signals could also arise by store operated $\mathrm{Ca}^{2+}$ entry (SOCE) through $\mathrm{Ca}^{2+}$ release-activated $\mathrm{Ca}^{2+}$ (CRAC) channels, which regulate signaling pathways essential for T-cell activation ${ }^{36}$, though these channels have been shown to have a minimal role in many macrophage functions ${ }^{37}$. Our observations that Piezo1 potentiates NFKB and mitigates STAT6 is consistent with reports that show increased intracellular $\mathrm{Ca}^{2}$ activates $\mathrm{NF \kappa B}$ and suppresses STAT6 in immune cells through $\mathrm{Ca}^{2+}$-dependent molecules such 


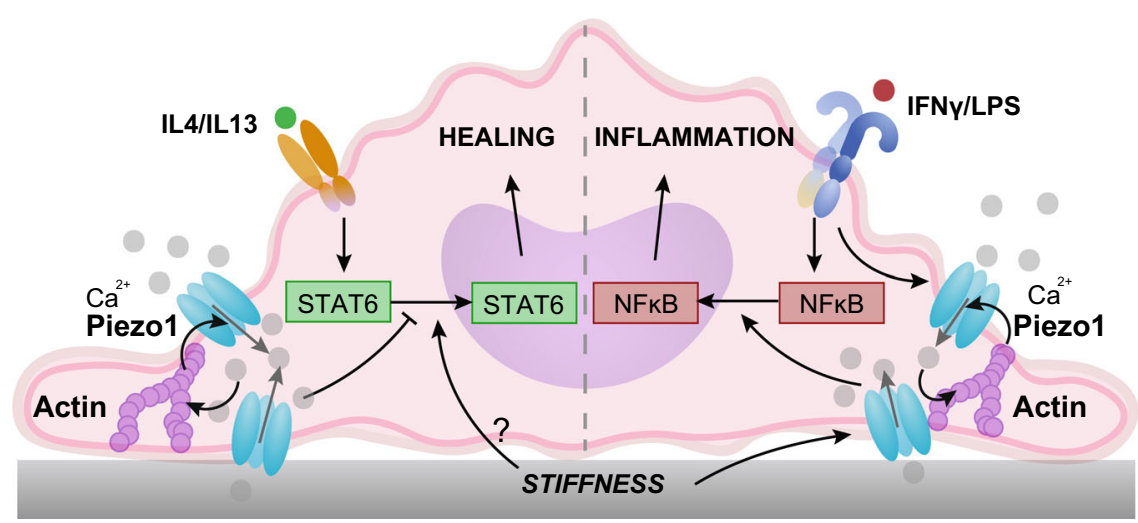

Fig. 6 Stiffness-dependent modulation of Piezo1 activity modulates macrophage activation. Activation of Piezo1 by IFN $\gamma /$ LPS on stiff substrates promotes actin polymerization, which enhances channel mediated $\mathrm{Ca}^{2+}$ influx. Positive regulation between actin and Piezo1 enhances inflammation

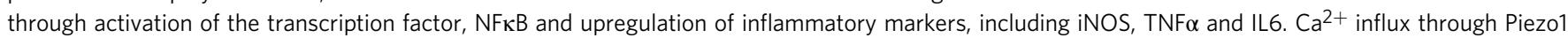
channels on stiff substrates inhibits the activation of the transcription factor STAT6 and expression of healing markers, such as ARG1. Other stiffness mediated and Piezo1 independent mechanisms likely upregulate STAT6 activation in vitro.

as calpains ${ }^{25,43}$. Furthermore, we show that pharmacological inhibition of calpains reduces inflammatory activation in macrophages, as has previously been shown by others ${ }^{48,49}$. Therefore, Piezol-mediated $\mathrm{Ca}^{2+}$ influx could potentially activate calpains resulting in enhanced inflammatory and suppressed healing activation phenotypes in macrophages.

We also show that Piezol plays a role in sensing environmental stiffness in macrophages. Stiff substrates have been shown to promote macrophage inflammatory activation through MyD88dependent pathways and enhanced $N F \kappa B$ activation 9,58 , but the role of stiffness in regulating healing activation has been less clear. We demonstrate stiffness-dependent increases in $\mathrm{Ca}^{2+}$ influx in response to the Piezo1 agonist Yoda1 and IFN $\gamma / \mathrm{LPS}$ stimulation. We found that activation of both inflammatory and healing pathways increased as substrate stiffness increased, suggesting that stiffness does not enhance inflammation at the expense of healing, but instead promotes overall macrophage responses to soluble cues. In addition, while inflammation scaled with stiffness, healing responses did not. This could potentially be attributed to the fact that macrophages cultured on 20 and $280 \mathrm{kPa}$ surfaces have enhanced cell spreading and healing activation compared to cells cultured on 1 and $40 \mathrm{kPa}$ surfaces, given that cell shape is known to play an important role in regulating macrophage activation $^{34,52}$. Moreover, stiffness-dependent increases in inflammatory activation required Piezo1, but depletion of Piezo1 reduced inflammation while enhancing healing pathways. Piezo1mediated $\mathrm{Ca}^{2+}$ influx in cells cultured on stiff substrates inhibits IL4/IL13 induced activation of the transcription factor STAT6, but other stiffness-mediated, Piezol independent mechanisms could influence STAT6 activation (Fig. 6). Pharmacological inhibition of STAT6 in cells seeded on 1 and $280 \mathrm{kPa}$ substrates revealed suppression of healing activation in cells cultured on both stiffness surfaces, which suggests that STAT6 signaling is prominent even on soft surfaces. Together, our data show that Piezol-mediated $\mathrm{Ca}^{2+}$ signaling promotes inflammation in stiff environments, but the regulation of healing pathways by stiffness and Piezol may be more complex.

The activity of mechanically activated ion channels such as Piezol has been shown to be dependent on external mechanical cues as well as internal cell-generated forces, and the cytoskeleton is pivotal in transducing such mechanical stimuli as well as generating membrane tension required for channel activity $^{17,47,59}$. We show that positive feedback regulation between Piezol and the actin cytoskeleton promotes macrophage inflammatory activation. Specifically, we find that Piezo1 enhances F-actin formation, consistent with what has been observed in lymphatic endothelial cells ${ }^{51}$, and that actin polymerization promotes Piezo1-mediated $\mathrm{Ca}^{2+}$ activity, suggesting a potential positive feedback regulation between ion channel activity and the cytoskeleton (Fig. 6). Our results also show that inhibition of F-actin with LatA suppresses inflammation, and stabilization of F-actin with Jasp enhances inflammation, consistent with what has been observed by others ${ }^{52,53}$. In addition, Jasp likely enhances other $\mathrm{Ca}^{2+}$ sources resulting in increased inflammatory responses in both control and macrophages lacking Piezol. Actin is known to regulate several TRP channels which could potentially explain this increase in non-Piezol mediated $\mathrm{Ca}^{2+}$ influx and enhanced inflammation from Jasp treatment ${ }^{60}$. Together, our results suggest that positive regulation between actin and Piezol enhances inflammatory activation in macrophages.

Mechanical cues are altered in the context of surgical implants, as well as during the development and progression of many pathological conditions in which macrophages are involved, including various forms of cancer, cardiovascular diseases, and fibrosis ${ }^{10,13,14}$. Our implant studies reveal that Piezo1 is required for macrophage sensing of different stiffness environments in vivo, and that its activity promotes inflammation and suppresses healing function of macrophages. The biomaterials used here allowed comparison of the immune response between soft materials that are similar in stiffness to tissues and the response to considerably stiffer materials that could represent biomedical implants ${ }^{11}$. Heightened stiffness has been associated with increased inflammatory activation in cardiovascular disease and healing activation in cancer and fibrosis ${ }^{14,61-64}$, and the more subtle changes in tissue stiffness in such contexts as well as disease models will need to be examined in future studies. Nonetheless, our study identifies a role for the mechanosensitive ion channel Piezol in sensing environmental stiffness in myeloid cells and influencing the foreign body response to implanted materials. Further exploring the role of Piezol in the context of disease will improve our understanding of the development of various pathological states and the role of macrophages in health and disease.

\section{Methods}

Animals. Generation of Piezo1 ${ }^{\Delta L y s M}$ mice was accomplished through breeding Piezo1 flox/flox (Jackson Laboratories stock no. 029213) and LysMCre/Cre (Jackson Laboratories stock no. 004781) mice together to generate progeny that were heterozygous for both genes. The generated heterozygotes were then bred with 
Piezol flox/flox mice to generate Piezol ${ }^{\text {flox/flox }} \mathrm{LysM}^{\mathrm{Cre} /+}\left(\mathrm{Piezo}^{\Delta L y s M}\right)$ and Pie$\mathrm{zol}^{\mathrm{flox} /+} \mathrm{LysM}^{\mathrm{Cre} /+}\left(\right.$ Piezo $\left.1^{f l /+}\right)$ mice. A similar breeding scheme was utilized to generate LSL-Salsa6f-Vav1 $1^{\mathrm{Cre} /+}$ mice used for calcium imaging. Piezo1 ${ }^{\text {Pl-tdT }}$ mice (Jackson Laboratories stock no. 029214) were obtained from the Pathak laboratory. Wild type C57BL/6 J mice (Jackson Laboratories) were also used in experiments that did not require genetic manipulation. Experiments were performed using animals of similar age and littermates were used as controls. Mice were maintained at $12 \mathrm{~h}$ light/dark cycles within temperature $\left(70-74^{\circ} \mathrm{F}\right)$ and humidity $(30-70 \%)$ controlled rooms. All animal experiments were performed in compliance with the University of California, Irvine's Institutional Animal Care and Use Committee under protocol \# AUP-20-047.

Cell isolation and culture. Bone-marrow-derived macrophages (BMDMs) were harvested from the femurs of 6-12-week-old C57BL/6 J mice. Bone marrow cells were collected by flushing the bone marrow of the femur with DMEM supplemented with $10 \%$ heat-inactivated FBS, $2 \mathrm{mM} \mathrm{L-glutamine,} 1 \%$ penicillin/streptomyocin (all from Thermo Fisher), and a 10\% conditioned media produced from CMG 14-12 cells expressing recombinant mouse macrophage colony stimulating factor (MCSF), which induces differentiation of bone marrow cells to macrophages. To remove red blood cells, the collected bone marrow cells were treated with a red cell lysis buffer, and then centrifuged before being resuspended in the previously mentioned media. After 7 days, the cells were harvested using an enzyme-free dissociation buffer (Fisher Scientific) and seeded onto surfaces that were coated with a $10 \mu \mathrm{g} / \mathrm{mL}$ fibronectin (Corning) solution. BMDMs were seeded at a density of $\sim 3.9 \times 10^{4}$ cells $/ \mathrm{cm}^{2}$ and were incubated overnight prior to stimulation with media (Unstim.), $0.3 \mathrm{ng} / \mathrm{mL}$ IFN $\gamma / \mathrm{LPS}$ or $0.1 \mathrm{ng} / \mathrm{mL}$ IL4/IL13. Following stimulation, cells were incubated for 1 or $18 \mathrm{~h}$ prior to collection. Peritoneal macrophages were harvested following established protocols ${ }^{65}$. Briefly, cold PBS was injected and retrieved from the peritoneal cavity of mice. The resulting cell suspension was centrifuged and resuspended in DMEM supplemented with $10 \%$ heat-inactivated FBS, $2 \mathrm{mM}$ L-glutamine, $1 \%$ penicillin/streptomyocin (all from Thermo Fisher). For studies involving pharmacological manipulation of the cytoskeleton, cells were incubated with either DMSO, $500 \mathrm{nM}$ latrunculinA (LatA), $500 \mathrm{nM}$ jasplakinolide (Jasp), or $25 \mu \mathrm{M}$ ML7 for an hour prior to stimulation with $0.3 \mathrm{ng} / \mathrm{mL}$ IFN $\gamma / \mathrm{LPS}$ for an additional $6 \mathrm{hrs}$.

Polyacrylamide gel fabrication. Polyacrylamide hydrogels of varying stiffness were fabricated using protocols previously described ${ }^{66}$. Briefly, cover slips were cleaned with $70 \%$ ethanol and dried prior to $10 \mathrm{~min}$ UVO treatment. The cover slips were then treated with bind-silane (solution containing 95 of $95 \%$ ethanol, $0.3 \%$ 3-(Trimethoxysilyl) propylmethacrylate, and 5 of $10 \%$ acetic acid) and were incubated for $5 \mathrm{mins}$ at room temperature. The coverslips were then washed with ethanol prior to incubation at $70^{\circ} \mathrm{C}$ for one hour. Meanwhile, glass slides were treated with silanization solution I and incubated in a vacuum desiccator for 5 mins. Glass slides were washed with DI water and blotted dry prior to gel formation. Solutions containing varying ratios of acrylamide:bis-acrylamide were pipetted onto the glass slides and the coverslip was placed onto the solution such that the polyacrylamide gel would be sandwiched between the bind-silane treated coverslip and salinization solution I treated glass slide. Gels were allowed to polymerize for $30 \mathrm{mins}$ and were then removed from the glass slide and placed into culture plates. The resulting hydrogels were conjugated with $20 \mu \mathrm{g} / \mathrm{ml}$ of fibronectin using sulfo-SANPAH (Thermo scientific) overnight at $4{ }^{\circ} \mathrm{C}$.

\section{$\mathrm{Ca}^{2+}$ imaging and analysis}

Confocal Imaging. BMDMs from LSL-Salsa6f-Vav1 $\mathrm{Cre} /+$ mice were seeded on fibronectin coated $35 \mathrm{~mm}$ MatTek dishes. Confocal imaging of $\mathrm{Ca}^{2+}$ dynamics in Salsa6f macrophages was accomplished using an Olympus Fluoview FV3000RS confocal laser scanning microscope which is equipped with a high-speed resonance scanner and IX3-ZDC2 Z-drift compensator. Cells were maintained at $37^{\circ} \mathrm{C}$ using the Tokai Hit incubation stage, excited using sequential line scan at $488 \mathrm{~nm}$ and $561 \mathrm{~nm}$ and imaged using an Olympus 40x silicone oil objective (NA 1.25). Ratiometric analysis of $\mathrm{Ca}^{2+}$ signals was performed through using ImageJ software. Briefly, Green (GCaMP6f) and Red (tdTomato) channel time-lapse images were converted to tiff files and background subtracted. ROIs were drawn around whole cells or individual $\mathrm{Ca}^{2+}$ events were outlined, and mean pixel intensities were obtained over time for both the $\mathrm{Ca}^{2+}$ independent tdTomato and the green $\mathrm{Ca}^{2+}$ sensitive GCaMP6f. Of note, ROIs were drawn around whole cells to quantify responses to Yodal, which produced global increases in cytosolic $\mathrm{Ca}^{2+}$ at the doses used in our study. In contrast, BMDMs exhibited $\mathrm{Ca}^{2+}$ events that were often restricted to cell processes or specific regions of the cell at baseline and after the addition of soluble signals, which necessitated drawing of ROIs around individual events for the purpose of quantification. Finally, a ratio of GCaMP6f to tdTomato mean intensities (G/R ratio) was calculated for each ROI and time point to generate $\mathrm{Ca}^{2+}$ traces. Peak intensities were obtained through finding the maximum $\mathrm{G} / \mathrm{R}$ ratio over time across individual cell or ROI. For quantification of responses to soluble signals, individual $\mathrm{Ca}^{2+}$ events were outlined as described above. The resulting $\mathrm{G} / \mathrm{R}$ ratios were then obtained, and the number of events computed through the use of a MATLAB script. Briefly, a polynomial fit was used to compute and subtract baseline values from $G / R$ ratios over time. This was followed by using a Gaussian filter to smooth the data before using a threshold-based analysis to identify the number of peaks within a signal, which corresponds to the number of $\mathrm{Ca}^{2+}$ events. The total number of events was normalized to the number of cells and time of acquisition. The total $\%$ active cells was obtained by dividing cells with one or more $\mathrm{Ca}^{2+}$ events to the total number of cells present in the field of view.

TIRF imaging. BMDMs from LSL-Salsa6f-Vav $1^{\mathrm{Cre} /+}$ mice were seeded on fibronectin coated $35 \mathrm{~mm}$ MatTek dishes. Imaging of $\mathrm{Ca}^{2+}$ dynamics in Salsa6f + macrophages was accomplished using an Olympus IX83 microscope that was equipped with an automated 4-line cellTIRF illuminator and a PLAPO 60x oil immersion objective (numerical aperture 1.45). Cells were illuminated with 488 and $561 \mathrm{~nm}$ lasers and images were acquired with Hamamatsu Flash $4.0 \mathrm{v} 2+$ scientific CMOS cameras at a $100 \mathrm{~Hz}$ frame rate. Analysis of the videos was accomplished using Flika, an open-source image analysis package, as previously mentioned 33

RNA interference. For experiments involving the reduction of PIEZO1 expression, unstimulated macrophages were exposed to non-target and PIEZO1 siRNA (both Dharmacon) in a Nucleofector ${ }^{\circledR}$ solution obtained from a primary cell 4DNucleofector ${ }^{\oplus}$ kit (Lonza). Following transfection, the cells were supplemented with warm media before being seeded onto experimental substrates. The transfected cells were allowed to adhere for $72 \mathrm{~h}$ prior to stimulation for an additional $18 \mathrm{~h}$.

Western blotting. BMDMs were rinsed with PBS before being exposed to a lysis buffer, a combination of RIPA lysis buffer and $1 \%$ protease inhibitor (both from Fisher Scientific). The substrates were scraped to release the adhered cells and the lysate was collected. The lysate was spun at $16000 \mathrm{~g}$ for $15 \mathrm{~min}$ and the supernatant was obtained. The proteins were denatured through the use of a Laemmli buffer supplemented with $5 \% 2$-mercaptoethanol at $95^{\circ} \mathrm{C}$ for 10 min before each sample was loaded into a well of a $4-15 \%$ mini-PROTEAN ${ }^{\mathrm{TM}}$ precast gel (all from Biorad). Gel electrophoresis resulted in the separation of proteins before being transferred onto nitrocellulose membranes using the iBlot dry blotting system (Thermo Fisher Scientific). Following electroblotting, the membranes were blocked using $5 \%$ nonfat milk in TBST overnight at $4{ }^{\circ} \mathrm{C}$. After 30 min of washing in TBST, the membranes were probed with a primary antibody (see Supplementary table 1 for list of antibodies used) for $1 \mathrm{~h}$ at room temperature. An additional $30 \mathrm{~min}$ of washing in TBST followed before the membranes were probed with secondary antibodies at room temperature for $1 \mathrm{~h}$. The membrane was then washed in TBST and immersed into a chemiluminescent HRP substrate solution (Thermo Scientific) and imaged using a ChemiDoc XRS System (Biorad). Uncropped blots are provided in the Source Data file.

ELISA. Following $18 \mathrm{~h}$ of stimulation, the supernatants were collected and analyzed for the presence of TNF- $\alpha$, IL- 6 , and MCP-1 using ELISA kits (BioLegend). The assays were conducted following the manufacturer's instructions.

RNA isolation and qPCR. Tri-reagent (Sigma) was added to samples to lyse cells and RNA isolation was performed following the manufacturer's instructions. cDNA was made using a cDNA reverse transcription kit (Applied Biosciences) and qPCR was performed using PerfeCTa ${ }^{\circ}$ SYBR $^{\oplus}$ Green SuperMix (QuantaBio), see Supplementary Table 2 for a list of primers used. All assays were performed in accordance with manufacturer's instructions.

Immunofluorescence. Following stimulation, BMDMs were fixed in $4 \%$ paraformaldehyde for a period of 10 mins. The fixed cells were washed in PBS prior to permeabilization in $0.1 \%$ or $0.3 \%$ Triton-X in PBS for staining of PIEZO1 ${ }^{\mathrm{P} 1-\text { tdT }}$ or NFkB/STAT6, respectively. Following additional PBS washes the cells were blocked in $2 \%$ BSA prior to being incubated with primary antibodies for $1 \mathrm{~h}$ at room temperature or overnight at $4^{\circ} \mathrm{C}$ (see Supplementary table 1 for list of antibodies used). The cells were then repeatedly washed with $2 \% \mathrm{BSA}$ and incubated with secondary antibodies in $2 \% \mathrm{BSA}$, for $1 \mathrm{~h}$ at room temperature. After repeated washing with 2\% BSA, the cells were incubated with Alexa Fluor 488 phalloidin (Fisher Scientific), diluted 1:100 in PBS, and Hoechst (Invitrogen), diluted 1:2000 in PBS, for $30 \mathrm{~min}$ at room temperature. The cells were thoroughly washed with PBS, before being mounted onto a glass slide and imaged using a Zeiss LSM700 confocal microscope or an Olympus Fluoview FV3000 confocal laser scanning microscope. Approximately 50 cells in each condition were outlined per experiment and the mean intensity or total intensity was computed for each cell using ImageJ.

Subcutaneous implant animal studies. Varying stiffness PEGDA gels were implanted into 6-week-old Piezo $1^{\Delta L y s M}$ and control Piezo $1^{f l /+}$ mice. Stiff $(\sim 140$ $\mathrm{kPa})$ and Soft $(\sim 1 \mathrm{kPa})$ PEGDA $400 \mathrm{MW}$ (Polysciences Inc.) were reconstituted in PBS at $50 \%$ or $10 \% \mathrm{w} / \mathrm{v}$, respectively, with $0.005 \%$ Irgacure 2959 photoinitiator. 1 $\mathrm{mm}$ sheets of hydrogels were cast and crosslinked using UV for $5 \mathrm{~min}$ and were cut into disks using $5 \mathrm{~mm}$ biopsy punches. Prior to implantation, mice were anesthetized using isoflurane and the dorsal skin was shaved and cleansed using $70 \%$ ethanol. Subcutaneous pockets were created on either side of a $\sim 5 \mathrm{~mm}$ incision 
along the dorsal midline, and soft and stiff PEGDA gels were placed inside, one on each side. The incision was closed using staples. Mice were housed individually after wounding and monitored daily for signs of infection/healing. Following 3 days, mice were sacrificed, and the implants were retrieved with surrounding tissue and mounted in OCT for cryosectioning.

Immunohistochemistry and histology. Frozen tissue sections were thawed and allowed to equilibrate to room temperature, fixed in $4 \%$ paraformaldehyde for 15 min, and then washed 4 times with PBS. Tissues were permeabilized using $0.2 \%$ Triton- $\mathrm{x}-100$ in PBS (Sigma) followed by three washes with $0.1 \%$ Tween-20 in PBS (Sigma), before blocking in 1\% BSA $+0.1 \%$ Tween in PBS for $2 \mathrm{~h}$. Sections were incubated in primary antibody overnight at $4{ }^{\circ} \mathrm{C}$ (see Supplementary table 1 for list of antibodies used). They were then washed three times and stained with a secondary antibody for $1 \mathrm{~h}$. Following three more washes slides were mounted with Fluoromount $\mathrm{G}$ and imaged at 20x using the Olympus FV3000 laser scanning confocal microscope. Images were analyzed using ImageJ software to quantify percent positive staining. Hoechst staining was used to create a nuclear mask and compute the total number of cells in each frame. $\mathrm{F} 4 / 80$ positively stained cells were counted and divided by the total number of cells to get $\% \mathrm{~F} 4 / 80+$ cells. In addition, to analyze macrophage-specific phenotypes, the number of cells positively stained for inflammatory and/or healing markers were divided by the total number of F4/ $80+$ cells. H\&E and Masson's trichrome staining was performed by the University of California, Irvine Research Services Core Facility and were imaged using a Nikon. Images were analyzed using ImageJ software to quantify the average thickness of the collagen capsule from the material/tissue interface to the start of the muscle layer. Images were also analyzed to quantify the number of cells within a given area to assess immune cell infiltration.

Statistics and reproducibility. Data are presented as the mean \pm standard deviation across at least three independent experiments. Representative images are accompanied with quantification from a minimum of three independent experiments. Comparisons were performed using a two-tailed Student's $t$ test, two-tailed paired $t$ test, or two-tailed Mann-Whitney $U$ test, as indicated in figure legends, and ${ }^{*} p<0.05$ was considered significant. Exact $p$-values are provided in the Source Data file.

Reporting summary. Further information on research design is available in the Nature Research Reporting Summary linked to this article.

\section{Data availability}

All relevant data supporting the key findings of this study are available within the article and its Supplementary Information files or from the corresponding author upon reasonable request. Source data are provided with this paper. A reporting summary for this article is available as a Supplementary Information file. Source data are provided with this paper.

\section{Code availability}

Flika, an open-source image processing and analysis package, can be obtained from: https://github.com/flika-org/flika.

Received: 16 November 2020; Accepted: 16 April 2021; Published online: 31 May 2021

\section{References}

1. Biswas, S. K. \& Mantovani, A. Macrophage plasticity and interaction with lymphocyte subsets: cancer as a paradigm. Nat. Immunol. 11, 889-896 (2010).

2. Mosser, D. M. \& Edwards, J. P. Exploring the full spectrum of macrophage activation. Nat. Rev. Immunol. 8, 958 (2008).

3. Sica, A. \& Mantovani, A. Macrophage plasticity and polarization: in vivo veritas. J. Clin. Invest. 122, 787-795 (2012).

4. Li, J., Chen, J. \& Kirsner, R. Pathophysiology of acute wound healing. Clin. Dermatol. 25, 9-18 (2007)

5. Hsieh, J. Y. et al. Differential regulation of macrophage inflammatory activation by fibrin and fibrinogen. Acta Biomater. 47, 14-24 (2017).

6. Sager, H. B. et al. Proliferation and recruitment contribute to myocardial macrophage expansion in chronic heart failure. Circulation Res. 119, https:// doi.org/10.1161/CIRCRESAHA.116.309001 (2016).

7. Meli, V. S. et al. Biophysical regulation of macrophages in health and disease. J. Leukoc. Biol. 106, 283-299 (2019).

8. Jain, N., Moeller, J. \& Vogel, V. Mechanobiology of macrophages: how physical factors coregulate macrophage plasticity and phagocytosis. Аnnu. Rev. Biomed. Eng. 21, 267-297 (2019).
9. Previtera, M. L. \& Sengupta, A. Substrate stiffness regulates proinflammatory mediator production through TLR4 activity in macrophages. PLOS ONE 10, e0145813 (2015).

10. Blakney, A. K., Swartzlander, M. D. \& Bryant, S. J. The effects of substrate stiffness on the in vitro activation of macrophages and in vivo host response to poly(ethylene glycol)-based hydrogels. J. Biomed. Mater. Res. A 100, 1375-1386 (2012)

11. Moshayedi, P. et al. The relationship between glial cell mechanosensitivity and foreign body reactions in the central nervous system. Biomaterials 35, 3919-3925 (2014)

12. Jansen, L. E. et al. Zwitterionic PEG-PC hydrogels modulate the foreign body response in a modulus-dependent manner. Biomacromolecules 19, 2880-2888 (2018).

13. Huang, S. \& Ingber, D. E. Cell tension, matrix mechanics, and cancer development. Cancer Cell 8, 175-176 (2005).

14. Tracqui, P. et al. Mapping elasticity moduli of atherosclerotic plaque in situ via atomic force microscopy. J. Struct. Biol. 174, 115-123 (2011).

15. Mobasheri, A., Carter, S. D., Martín-Vasallo, P. \& Shakibaei, M. Integrins and stretch activated ion channels; putative components of functional cell surface mechanoreceptors in articular chondrocytes. Cell Biol. Int. 26, 1-18 (2002).

16. Wang, J. H.-C. \& Thampatty, B. P. An introductory review of cell mechanobiology. Biomech. Model Mechanobiol. 5, 1-16 (2006).

17. Pathak, M. M. et al. Stretch-activated ion channel Piezol directs lineage choice in human neural stem cells. Proc. Natl Acad. Sci. USA 111, 16148-16153 (2014).

18. Chen, X. et al. A feedforward mechanism mediated by mechanosensitive ion channel PIEZO1 and tissue mechanics promotes glioma aggression. Neuron 100, 799-815.e7 (2018)

19. Blythe, N. M. et al. Mechanically activated Piezol channels of cardiac fibroblasts stimulate p38 mitogen-activated protein kinase activity and interleukin-6 secretion. J. Biol. Chem. https://doi.org/10.1074/jbc.RA119.009167 (2019).

20. Cahalan, S. M. et al. Piezol links mechanical forces to red blood cell volume. eLife 4, e07370 (2015).

21. Ranade, S. S. et al. Piezo1, a mechanically activated ion channel, is required for vascular development in mice. PNAS 111, 10347-10352 (2014).

22. Coste, B. et al. Piezol and Piezo2 are essential components of distinct mechanically activated cation channels. Science 330, 55-60 (2010).

23. Li, J. et al. Piezol integration of vascular architecture with physiological force. Nature 515, 279-282 (2014).

24. Jin, Y. et al. Functional role of mechanosensitive ion channel Piezol in human periodontal ligament cells. Angle Orthod. 85, 87-94 (2015).

25. Schappe, M. S. et al. Chanzyme TRPM7 mediates the Ca2+ influx essential for lipopolysaccharide-induced toll-like receptor 4 endocytosis and macrophage activation. Immunity 48, 59-74.e5 (2018).

26. Chauhan, A. et al. M1 macrophage polarization is dependent on TRPC1mediated calcium entry. iScience 8, 85-102 (2018).

27. Solis, A. G. et al. Mechanosensation of cyclical force by PIEZO1 is essential for innate immunity. Nature 573, 69-74 (2019).

28. Baratchi Sara et al. Transcatheter aortic valve implantation represents an antiinflammatory therapy via reduction of shear stress-induced, piezo1-mediated monocyte activation. Circulation 142, 1092-1105 (2020)

29. Aykut, B. et al. Targeting Piezol unleashes innate immunity against cancer and infectious disease. Science Immunology 5, eabb5168 (2020).

30. Goswami, R. et al. TRPV4 calcium-permeable channel is a novel regulator of oxidized LDL-induced macrophage foam cell formation. Free Radic. Biol. Med. 110, 142-150 (2017).

31. Link, T. M. et al. TRPV2 has a pivotal role in macrophage particle binding and phagocytosis. Nat. Immunol. 11, 232-239 (2010).

32. Chemaly, A. E., Nunes, P., Jimaja, W., Castelbou, C. \& Demaurex, N. Hv1 proton channels differentially regulate the $\mathrm{pH}$ of neutrophil and macrophage phagosomes by sustaining the production of phagosomal ROS that inhibit the delivery of vacuolar ATPases. J. Leukoc. Biol. 95, 827-839 (2014).

33. Maroto, R. et al. TRPC1 forms the stretch-activated cation channel in vertebrate cells. Nat. Cell Biol. 7, 179-185 (2005).

34. McWhorter, F. Y., Wang, T., Nguyen, P., Chung, T. \& Liu, W. F. Modulation of macrophage phenotype by cell shape. Proc. Natl Acad. Sci. USA 110, 17253-17258 (2013)

35. Dorrington, M. G. \& Fraser, I. D. C. NF-kB signaling in macrophages: dynamics, crosstalk, and signal integration. Front Immunol 10, 705 (2019).

36. Waqas, S. F. H., Ampem, G. \& Röszer, T. Analysis of IL-4/STAT6 Signaling in Macrophages. Methods Mol. Biol. 1966, 211-224 (2019).

37. Binnemars-Postma, K., Bansal, R., Storm, G. \& Prakash, J. Targeting the Stat6 pathway in tumor-associated macrophages reduces tumor growth and metastatic niche formation in breast cancer. FASEB J. 32, 969-978 (2018).

38. Xiao, H. et al. M2-like tumor-associated macrophage-targeted codelivery of STAT6 inhibitor and IKK $\beta$ siRNA induces M2-to-M1 repolarization for cancer immunotherapy with low immune side effects. ACS Cent. Sci. 6, $1208-1222(2020)$ 
39. $\mathrm{Yu}, \mathrm{T}$. et al. Modulation of M2 macrophage polarization by the crosstalk between Stat6 and Trim24. Nat. Commun. 10, 4353 (2019).

40. Czimmerer, Z. et al. The transcription factor STAT6 mediates direct repression of inflammatory enhancers and limits activation of alternatively polarized macrophages. Immunity 48, 75-90.e6 (2018).

41. Hoeksema, M. A. et al. IFN- $\gamma$ priming of macrophages represses a part of the inflammatory program and attenuates neutrophil recruitment. J. Immunol. 194, 3909-3916 (2015).

42. Bagaev, A. V. et al. Elevated pre-activation basal level of nuclear NF- $\kappa B$ in native macrophages accelerates LPS-induced translocation of cytosolic NF- $\mathrm{kB}$ into the cell nucleus. Sci. Rep. 9, 4563 (2019).

43. Zamorano, J., Rivas, M. D., Setien, F. \& Perez-G, M. Proteolytic regulation of activated STAT6 by calpains. J. Immunol. 174, 2843-2848 (2005).

44. Syeda, R. et al. Chemical activation of the mechanotransduction channel Piezol. Elife 4, e07369 (2015).

45. Dong, T. X. et al. T-cell calcium dynamics visualized in a ratiometric tdTomato-GCaMP6f transgenic reporter mouse. eLife 6, e32417 (2017).

46. Dong, T. X. et al. Intermittent $\mathrm{Ca} 2+$ signals mediated by Orail regulate basal T cell motility. Elife 6, e27827 (2017).

47. Ellefsen, K. L. et al. Myosin-II mediated traction forces evoke localized Piezo1dependent Ca 2+ flickers. Commun. Biol. 2, 1-13 (2019).

48. Griscavage, J. M., Wilk, S. \& Ignarro, L. J. Inhibitors of the proteasome pathway interfere with induction of nitric oxide synthase in macrophages by blocking activation of transcription factor NF-kappa B. Proc. Natl Acad. Sci. USA 93, 3308-3312 (1996).

49. Acharya, D., Li, X. R. (Lisa), Heineman, R. E.-S. \& Harrison, R. E. Complement receptor-mediated phagocytosis induces proinflammatory cytokine production in murine macrophages. Front. Immunol. 10, 3049 (2020).

50. Nourse, J. L. \& Pathak, M. M. How cells channel their stress: Interplay between Piezol and the cytoskeleton. Semin. Cell Dev. Biol. 71, 3-12 (2017).

51. Nonomura, K. et al. Mechanically activated ion channel PIEZO1 is required for lymphatic valve formation. Proc. NAS 115, 12817-12822 (2018).

52. Jain, N. \& Vogel, V. Spatial confinement downsizes the inflammatory response of macrophages. Nat. Mater. 17, 1134-1144 (2018).

53. Pergola, C. et al. Modulation of actin dynamics as potential macrophage subtype-targeting anti-tumour strategy. Sci. Rep. 7, 41434 (2017).

54. Date, D. et al. Kruppel-like transcription factor 6 regulates inflammatory macrophage polarization. J. Biol. Chem. 289, 10318-10329 (2014).

55. Liao, X. et al. Krüppel-like factor 4 regulates macrophage polarization. J. Clin. Invest. 121, 2736-2749 (2011).

56. Arranz, A. et al. Akt1 and Akt2 protein kinases differentially contribute to macrophage polarization. Proc. Natl Acad. Sci. USA 109, 9517-9522 (2012).

57. Murray, P. J. Macrophage polarization. Annu. Rev. Physiol. 79, 541-566 (2017).

58. Chen, M. et al. Substrate stiffness modulates bone marrow-derived macrophage polarization through NF- $\mathrm{kB}$ signaling pathway. Bioact. Mater. 5, 880-890 (2020).

59. Gottlieb, P. A., Bae, C. \& Sachs, F. Gating the mechanical channel Piezol. Channels 6, 282-289 (2012).

60. Smani, T., Dionisio, N., López, J. J., Berna-Erro, A. \& Rosado, J. A Cytoskeletal and scaffolding proteins as structural and functional determinants of TRP channels. Biochim. Biophys. Acta (BBA)-Biomembranes 1838, 658-664 (2014).

61. Emon, B., Bauer, J., Jain, Y., Jung, B. \& Saif, T. Biophysics of tumor microenvironment and cancer metastasis-a mini review. Comput. Struct. Biotechnol. J. 16, 279-287 (2018).

62. Mozos, I. et al. Inflammatory markers for arterial stiffness in cardiovascular diseases. Front Immunol 8, 1058 (2017).

63. Acerbi, I. et al. Human breast cancer invasion and aggression correlates with ecm stiffening and immune cell infiltration. Integr. Biol. (Camb.) 7, 1120-1134 (2015).

64. Smithmyer, M. E., Sawicki, L. A. \& Kloxin, A. M. Hydrogel scaffolds as in vitro models to study fibroblast activation in wound healing and disease. Biomater. Sci. 2, 634-650 (2014).

65. Zhang, X., Goncalves, R. \& Mosser, D. M. The isolation and characterization of murine macrophages. Curr. Protoc. Immunol. Chapter 14, Unit-14.1 (2008).

66. Tse, J. R. \& Engler, A. J. Preparation of hydrogel substrates with tunable mechanical properties. Curr. Protoc. Cell Biol. 47, 10.16.1-10.16.16 (2010).

\section{Acknowledgements}

This work was supported by the National Institutes of Health (NIH) National Institute of Allergy and Infectious Disease (NIAID) Grants R21AI128519-01 and R01AI151301, National Institute of Biomedical Imaging and Bioengineering (NIBIB) Grant R21EB027840-01, and National Institute of Arthritis, Musculoskeletal and Skin Diseases (NIAMS) Grant R21AR077288 to W.F.L.; NIAID R01AI121945 and National Institute of Neurological Disorders and Stroke (NINDS) R01NS14609 to M.D.C.; and NIH Director's Fund DP2AT010376 and NINDS R01NS109810 to M.M.P. H.A. was supported by NIH National Institute T32 Training Grant in Cardiovascular Applied Research and Entrepreneurship (5T32 HL116270-3) and American Heart Association Pre-Doctoral Fellowship (20PRE35200220), J.R.H. was supported by HHMI Gilliam Fellowship for Advanced Study awarded to J.R.H. and M.M.P. (GT11549), R.R.N. was supported by the UC Irvine Medical Scientist Training Program NIH training grant (T32 GM008620-18) and NIH NIAID F30 fellowship (AI142988-01A1). This study was made possible, in part, through access to the Optical Biology Core Facility of the Developmental Biology Center, a shared resource supported by the Cancer Center Support Grant (CA-62203) and Center for Complex Biological Systems Support Grant (GM-076516) at the University of California, Irvine. This work was also made possible, in part, through access to a confocal microscope within the Edwards Lifesciences Center for Advanced Cardiovascular Technology supported by (1S10OD025064-01Al) and the microscope imaging core within the Sue and Bill Gross Stem Cell Research Center at the University of California Irvine. We would like to acknowledge and thank Dr. Adeela Syed and Allia Fawaz for assistance with confocal microscopy as well as Andrew Phan, Hamid Abuwarda, Jessica Chin, Kevin Jiang, and Andrew Flach for their help with experiments.

\section{Author contributions}

H.A., V.S.M., M.D.C., M.M.P., and W.F.L. conceived the project and designed the experiments. H.A., V.S.M., P.K.V., K.T.B., and H.E.L. designed, performed, and analyzed macrophage functional experiments. H.A., A.J., and S.O. designed, performed, and analyzed confocal $\mathrm{Ca}^{2+}$ imaging studies. H.A. and J.R.H. designed, performed, and analyzed TIRF microscopy studies. H.A. and R.R.N. designed, performed, and analyzed implant studies. H.A., A.J., M.D.C., M.M.P., and W.F.L. wrote the manuscript.

\section{Competing interests}

The authors declare no competing interests.

\section{Additional information}

Supplementary information The online version contains supplementary material available at https://doi.org/10.1038/s41467-021-23482-5.

Correspondence and requests for materials should be addressed to W.F.L.

Peer review information Nature Communications thanks the anonymous reviewer(s) for their contribution to the peer review of this work. Peer reviewer reports are available.

Reprints and permission information is available at http://www.nature.com/reprints

Publisher's note Springer Nature remains neutral with regard to jurisdictional claims in published maps and institutional affiliations.

Open Access This article is licensed under a Creative Commons Attribution 4.0 International License, which permits use, sharing, adaptation, distribution and reproduction in any medium or format, as long as you give appropriate credit to the original author(s) and the source, provide a link to the Creative Commons license, and indicate if changes were made. The images or other third party material in this article are included in the article's Creative Commons license, unless indicated otherwise in a credit line to the material. If material is not included in the article's Creative Commons license and your intended use is not permitted by statutory regulation or exceeds the permitted use, you will need to obtain permission directly from the copyright holder. To view a copy of this license, visit http://creativecommons.org/ licenses/by/4.0/.

(c) The Author(s) 2021 